NISTIR 8158

\title{
Usability Testing of a Contactless Fingerprint Device: Part 1
}

Brian Stanton

Mary Theofanos

Susanne Furman

John M. Libert Shahram Orandi

John Grantham

This publication is available free of charge from: https://doi.org/10.6028/NIST.IR.8158

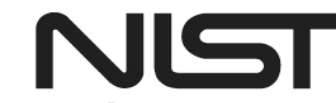

National Institute of Standards and Technology

U.S. Department of Commerce 
NISTIR 8158

\title{
Usability Testing of a Contactless Fingerprint Device: Part 1
}

\author{
Brian Stanton \\ Mary Theofanos \\ Susanne Furman \\ John M. Libert \\ Shahram Orandi \\ Information Access Division \\ Information Technology Laboratory \\ John Grantham \\ Systems Plus, INC.
}

This publication is available free of charge from:

https://doi.org/10.6028/NIST.IR.8158

December 2016

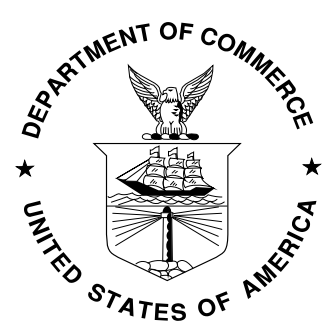

U.S. Department of Commerce

Penny Pritzker, Secretary

National Institute of Standards and Technology Willie May, Under Secretary of Commerce for Standards and Technology and Director 


\section{$\underline{\text { Table of Contents }}$}

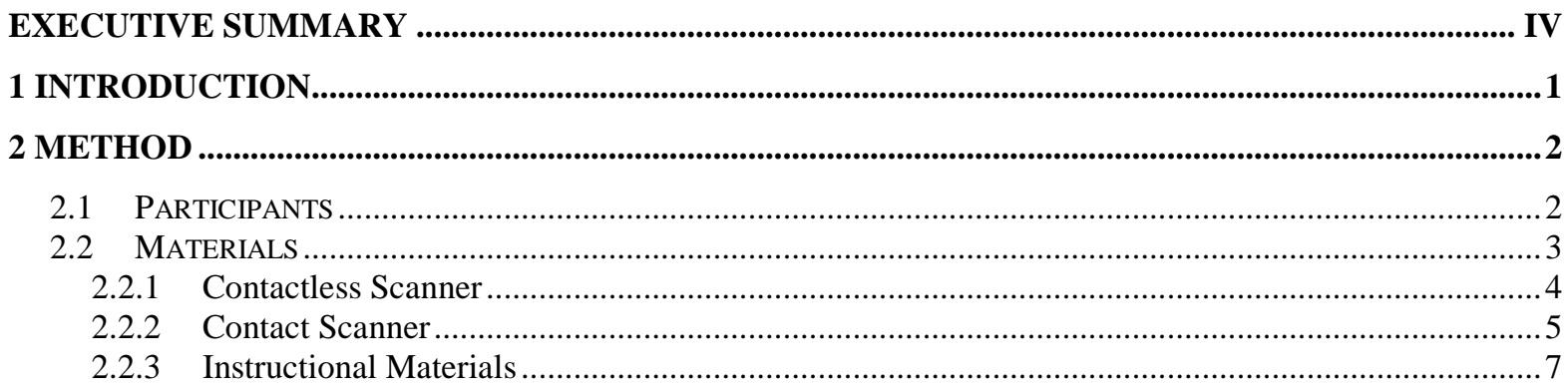

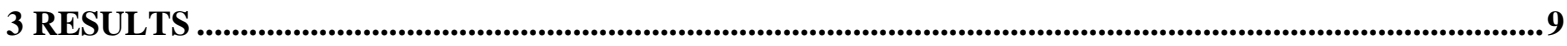

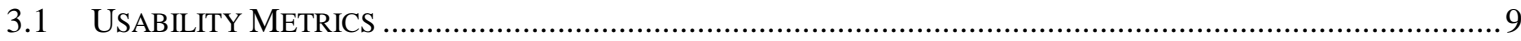

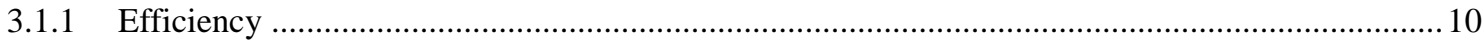

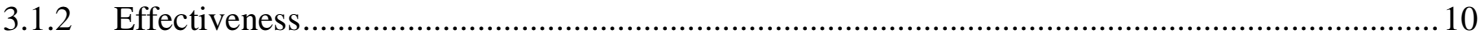

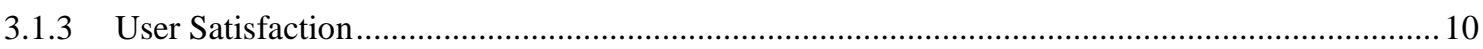

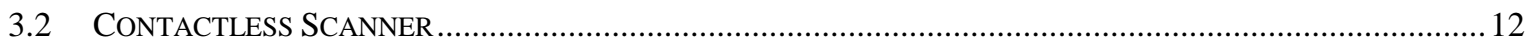

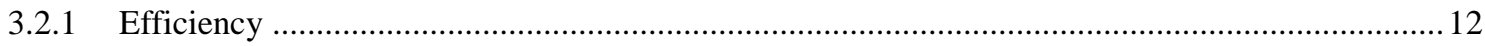

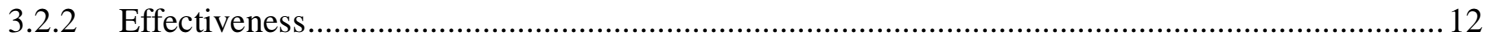

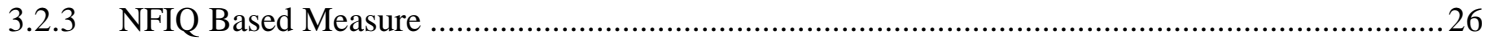

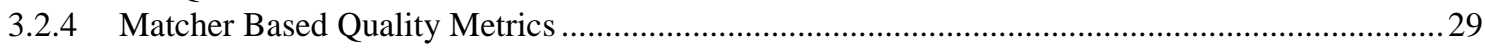

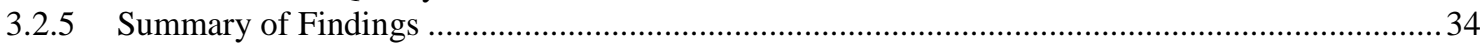

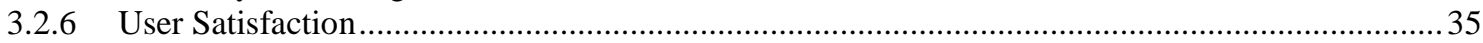

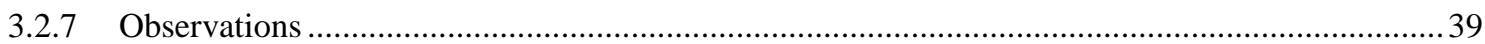

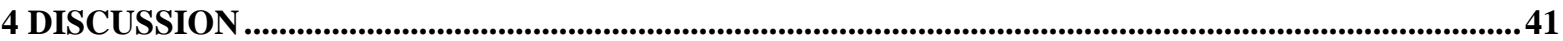

4.1 MENTAL MODELS OF FINGERPRINTING - TOUCHING THE GLASS ................................................41

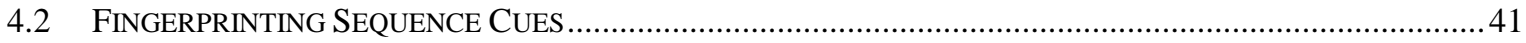

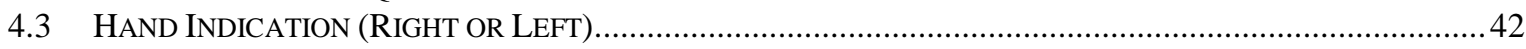

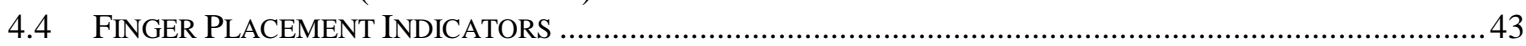

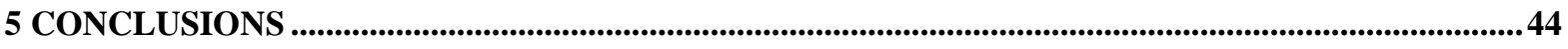

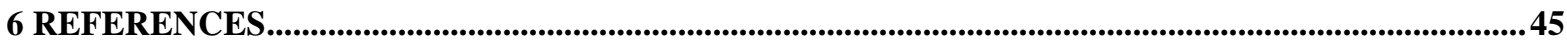

APPENDIX A: DEMOGRAPHICS QUESTIONNAIRE.......................................................................46

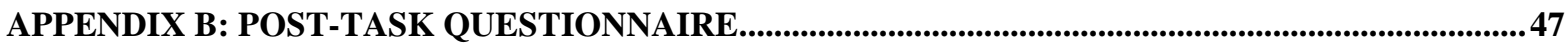




\section{List of Figures}

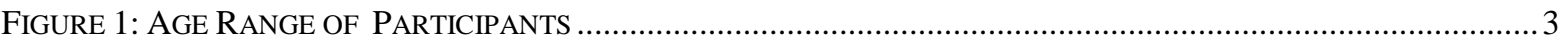

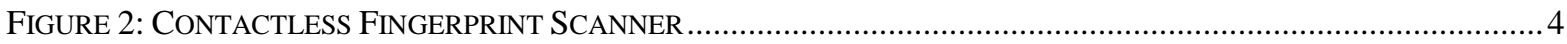

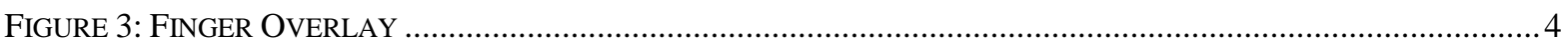

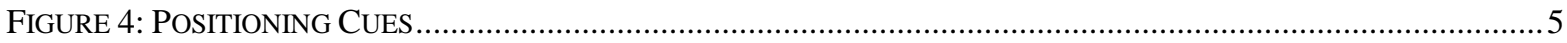

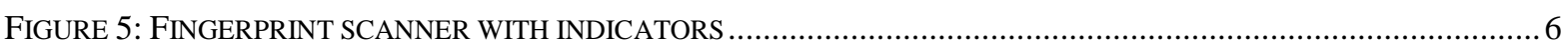

FigURE 7: FINGERPRINT SCANNER SETUP SHOWING MAT AND ADJUSTABLE TABLE ...........................................

FIGURE 14 DEMONSTRATION OF CONTRAST REVERSAL OF CONTACTLESS SCANNER............................................15

Figure 21 - BoXPlot OF NFIQ SCORES (CONTACTLESS SCANNER VS. CONTACT CONTROL SCANNER) ..............28

Figure 22 - Cumulative FMR/FNMR Probability GRAPH For ContaCt CONTROL SCANNER MATChES to

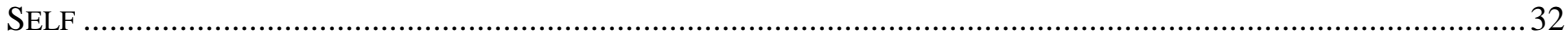

FigURE 23 - CUMULATIVE FMR/FNMR PROBABILITY GRAPH FOR CONTACTLESS SCANNER MATCHES TO SELF 32

Figure 24 - Cumulative FMR/FNMR PROBABILITY GRAPH FOR CONTACT CONTROL SCANNER MATCHES TO

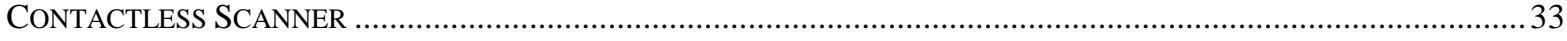

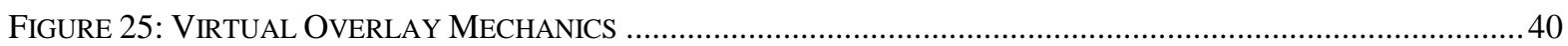

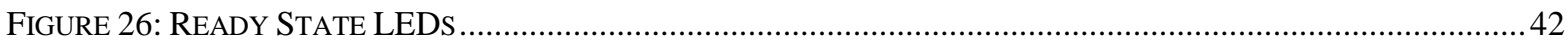

FiguRE 27: VIRTUAL OVERLAY AS SEEN THROUGH VIEWPORT .......................................................................... 43 


\section{$\underline{\text { List of Tables }}$}

TABLE 1: AVERAge TASK TIMES IN SECONDS - ConTACTLESS SCANNER …........................................................12

TABLE 2: SUCCESSFUl PRINT COLLECTION PER TASK - CONTACTLESS SCANNER ...............................................12

TABLE 3 - COUNT OF NFIQ SCORES FOR CONTACTLESS SCANNER AND CONTACT CONTROL SCANNER ................27

TABLE 4 - STATISTICAL COMPARISON OF NFIQ SCORES FROM CONTACTLESS SCANNER AND CONTACT CONTROL

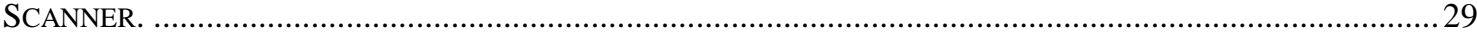

TABLE 5 - Match PERFoRmance SUMmaRy For IDENTIFICATION SCENARIOS ..................................................31

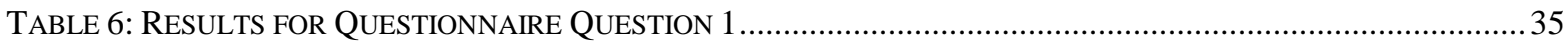

TABLE 7: RESULTS FOR QUESTIONNAIRE QUESTION 2 ..................................................................................... 36

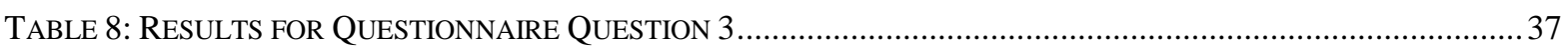

TABLE 9: RESULTS FOR QUESTIONNAIRE QUESTION 4................................................................................... 37

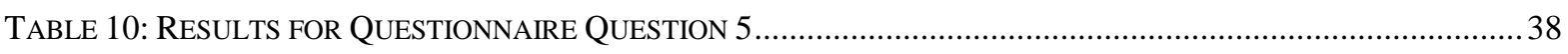

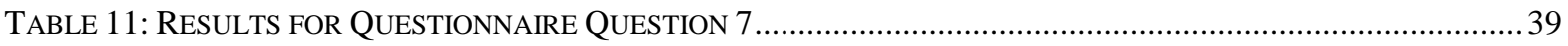




\section{EXECUTIVE SUMMARY}

The use of biometrics to identify individuals has become an important component of efforts to ensure U.S. national security, and has also grown rapidly. Biometrics are, for example, an integral part of the United States Visitor and Immigrant Status Indicator Technology (USVISIT) program. Utilization of biometrics in such systems was mostly a response to urgent security needs, and applications of such technologies were initially limited to state-ofindustry biometric collection devices.

Many risks involved in the operation of these systems have been identified and addressed. Some risks, however, simply cannot be addressed given the current system designs. To address these issues, DHS Science \& Technology (S\&T) commissioned and funded two projects to research technologies that could be used to acquire fingerprints without physical contact. While this novel approach to fingerprint capture solves several challenges posed by the previous generation of scanners, it also creates several new challenges that must be addressed.

Sixty-one participants volunteered for the study. Half of the volunteers started with a traditional contact scanner and the other half started with a prototype contactless scanner. They were asked to leave two sets of prints (the four fingers of each hand) three times for each scanner. First with no instructions, secondly after seeing an instructional video, and thirdly with verbal instructions. The participants then followed the same procedure with the second scanner. The order of scanners were counterbalanced across participants.

Overall the contactless scanner took longer to acquire the prints, acquired fewer prints under the no instruction and video instructional conditions, and was preferred less than the contact scanner.

Testing of images captured by the contactless scanner showed that these images can be assessed for quality, processed to detect minutiae and generate minutiae matching templates, and the resulting minutiae templates matched against other fingerprint minutiae templates. Minutiae templates are the currency for fingerprint matching, hence essential to the very function of such devices. Perhaps more importantly the extraction of minutiae is essential for assessing the interoperability of contactless devices with fingerprints collected using “legacy” fingerprint capture devices. 


\section{INTRODUCTION}

The United States Department of Homeland Security (DHS) is committed to using cuttingedge technologies and scientific talent in its mission to make America safer. The DHS Science and Technology Directorate (S\&T) is tasked with researching and organizing the scientific, engineering, and technological resources of the U.S. and leveraging these resources into technological tools to help protect the homeland. The Homeland Security Advanced Research Projects Agency (HSARPA) Biometrics Detector Program supports this effort.

The use of biometrics to identify individuals has become an important component of efforts to ensure U.S. national security, and has also grown rapidly. Biometrics are, for example, an integral part of the United States Visitor and Immigrant Status Indicator Technology (USVISIT) program. Utilization of biometrics in such systems was mostly a response to urgent security needs, and applications of such technologies were initially limited to state-ofindustry biometric collection devices. These devices included primarily optical and capacitive discharge capture equipment for contact-based electronic fingerprint collection, conducted in a manner similar to ink-based fingerprinting.

Many risks involved in the operation of these systems have been identified and addressed. Some risks, however, simply cannot be addressed given the current system designs. One such risk is the transmission of pathogens by the contact surface of the state-of-industry scanners. Another risk factor identified since wide deployment of biometric systems was the impact of the new biometric sample collection pathways on the existing daily operations: some new collection tasks added anywhere from 15 seconds to over a minute to the normal operations processes, depending on the number of samples being collected. In many cases, the additional time required for biometric collection significantly slowed the throughput of the overall process such collection was intended to support.

To address these issues, DHS S\&T commissioned and funded two projects to research technologies that could be used to acquire fingerprints without physical contact. These technologies utilize structured light illumination and optical spectrum with focus diversity. The goal of such a system is to develop the design basis for a whole new generation of biometric capture devices that can rapidly capture high-resolution images of all 10 fingerprints - again, without physical contact with the biometric sensor. While this novel approach to fingerprint capture solves several challenges posed by the previous generation of scanners, it also creates several new challenges that must be addressed.

Some of these challenges are related to human factors that affect biometric systems performance. It is necessary to closely examine these human factors issues by studying the usability of new contactless fingerprinting devices in terms of ergonomics and anthropometrics, affordance, accessibility, and user satisfaction of non-traditional form factors. Input on human factors and anthropometrics will result in a more robust operational 
system that increases user performance (timing and quality) and encourages user acceptance. This report describes part one of a two part report on the usability tests performed on two contactless prototypes by the National Institute of Standards and Technology (NIST) .

According to ISO 9241-11, usability is "the extent to which a product can be used by specified users to achieve specified goals with effectiveness, efficiency and satisfaction in a specified context of use” [5].

- Efficiency is a measure of the resources expended in relation to the accuracy and completeness with which users achieve goals. Efficiency is related to productivity and is generally measured as task time.

- Effectiveness is a measure of the accuracy and completeness with which users achieve specified goals. Common metrics include completion rate and number of errors.

- User Satisfaction is the degree to which the product meets the users' expectations-a subjective response in terms of ease of use, satisfaction, and usefulness.

This study measured the prototype contactless scanner on each of the three dimensions of usability.

\section{METHOD}

\subsection{PARTICIPANTS}

Sixty one NIST employees volunteered to participate in this study. The group consisted of a relatively equal number of men (33) and women (28), and the ages were fairly uniformly distributed, as seen in Figure 1. 


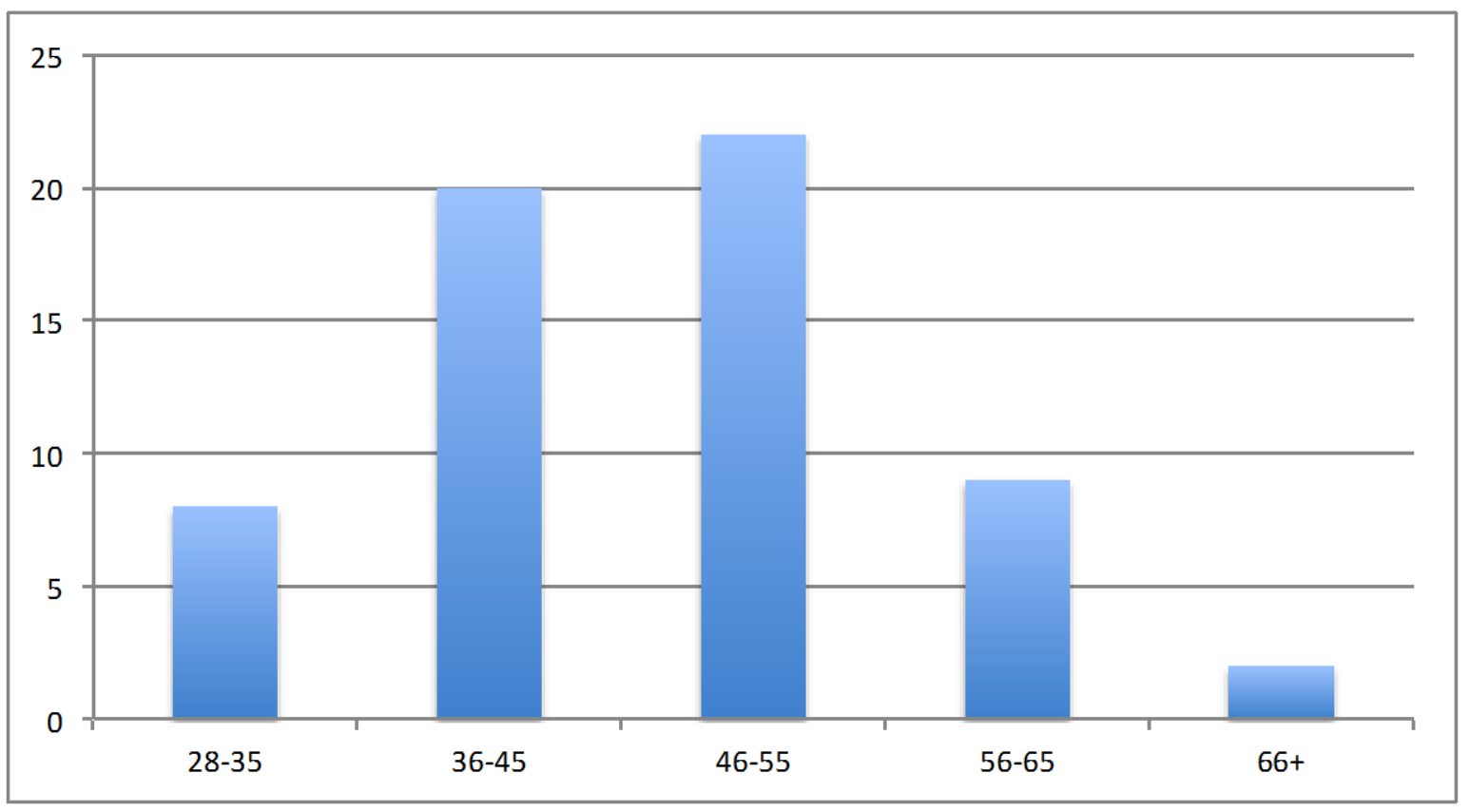

Figure 1: Age Range of Participants

85\% were right-handed and are very representative of the general population, $87 \%$ of which is right-handed [1].

\subsection{MATERIALS}

The materials for the test consisted of:

- A contactless fingerprint scanner ${ }^{1}$

- A traditional contact scanner that was positioned on a platform at 20 degrees

- Adjustable tables that allowed for accurate positioning of scanner height

- Floor mats with silhouettes of yellow feet to indicate where participants should stand

- Hand size estimator

- Video instructions

\footnotetext{
1 Specific hardware and software products identified in this report were used in order to perform the evaluations described. In no case does such identification imply recommendation or endorsement by the National Institute of Standards and Technology, nor does it imply that the products and equipment identified are necessarily the best available for the purpose.
} 
- Custom software

\subsubsection{Contactless Scanner}

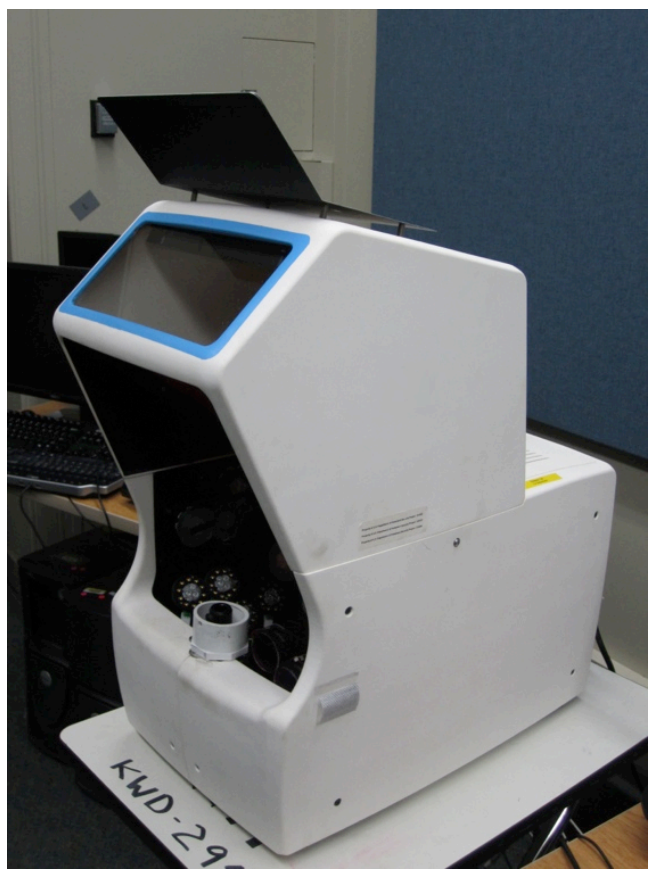

Figure 2: Contactless Fingerprint Scanner

The contactless fingerprint scanner used in this report measured $53.34 \mathrm{~cm}$ (21 in) $\mathrm{x} 33.02 \mathrm{~cm}$ (13 in) x $58.42 \mathrm{~cm}$ (23 in). It had a $12.70 \mathrm{~cm}$ (5 in) x $25.40 \mathrm{~cm}$ (10 in) viewing port (outlined in blue in the picture above) and a $33.02 \mathrm{~cm}$ (13 in) x $27.94 \mathrm{~cm}$ (11 in) opening in which participants were to hold their hand for scanning. To indicate that the tracking software was running, a picture of a right hand was displayed within the scanner. Once the device was ready to accept prints, the participants saw, through the viewport, a finger overlay with positioning cues (see Figure 3). The participants were to align their fingers with the overlay.

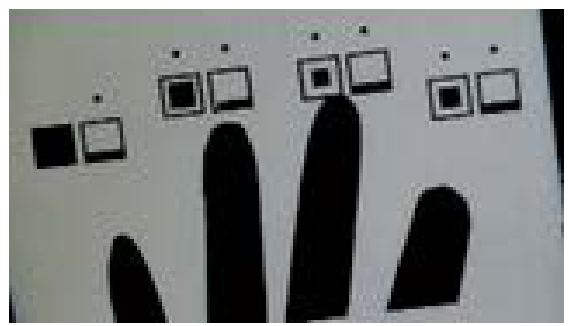

Figure 3: Finger Overlay 


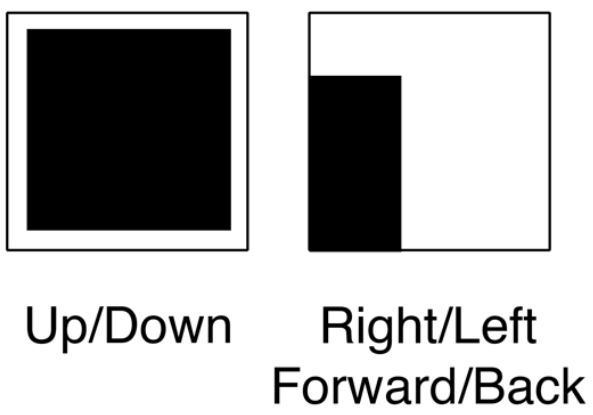

Figure 4: Positioning Cues

There were two positioning cues per finger. The first cue signaled the vertical positioning of the hand, and the second signaled right/left and forward/back for each finger, as shown in Figure 4. The black box within each square outline moved in concert with each finger, reflecting the finger's position within the scanning space by growing/shrinking (for first cue) or moving (for the second cue) to fill more of the outline as the finger approached the correct position. Once the squares associated with a finger were completely black - indicating that the finger was in the correct position - the print from that finger would be captured. The positioning cues for that finger would be replaced by a large oval within a box and then removed from the display.

\subsubsection{Contact Scanner}

The traditional contact fingerprint scanner used in this study - shown in Figure 5 - measured approximately $15.2 \mathrm{~cm}$ (6.0 in) x $15.2 \mathrm{~cm}$ (6.0 in) x $15.2 \mathrm{~cm}$ (6.0 in). On top of the scanner was a glass platen: this was the contact surface upon which participants placed their fingers for fingerprint capture. Above the platen was a line of four Light Emitting Diodes (LEDs) at the top edge of the scanner platen surface, each capable of emitting a red or a green light. On each side of the platen were two indicators (four in total) corresponding to a Right Slap, Right Thumb (on the right side), Left Slap, and Left Thumb (on the left side). These indicators would light up to indicate which fingers or thumb the participant was supposed to present. The scanner also emitted audible tones - beeps - whenever it successfully captured a print image. Note that what appears to be a horizontal line or delineation between the left and right icons is inside the scanner and not anything present at the surface of the platen. 
LEDs glowed red at the beginning of the process and when the participant's finger position was incorrect; they glowed green when finger positioning was correct

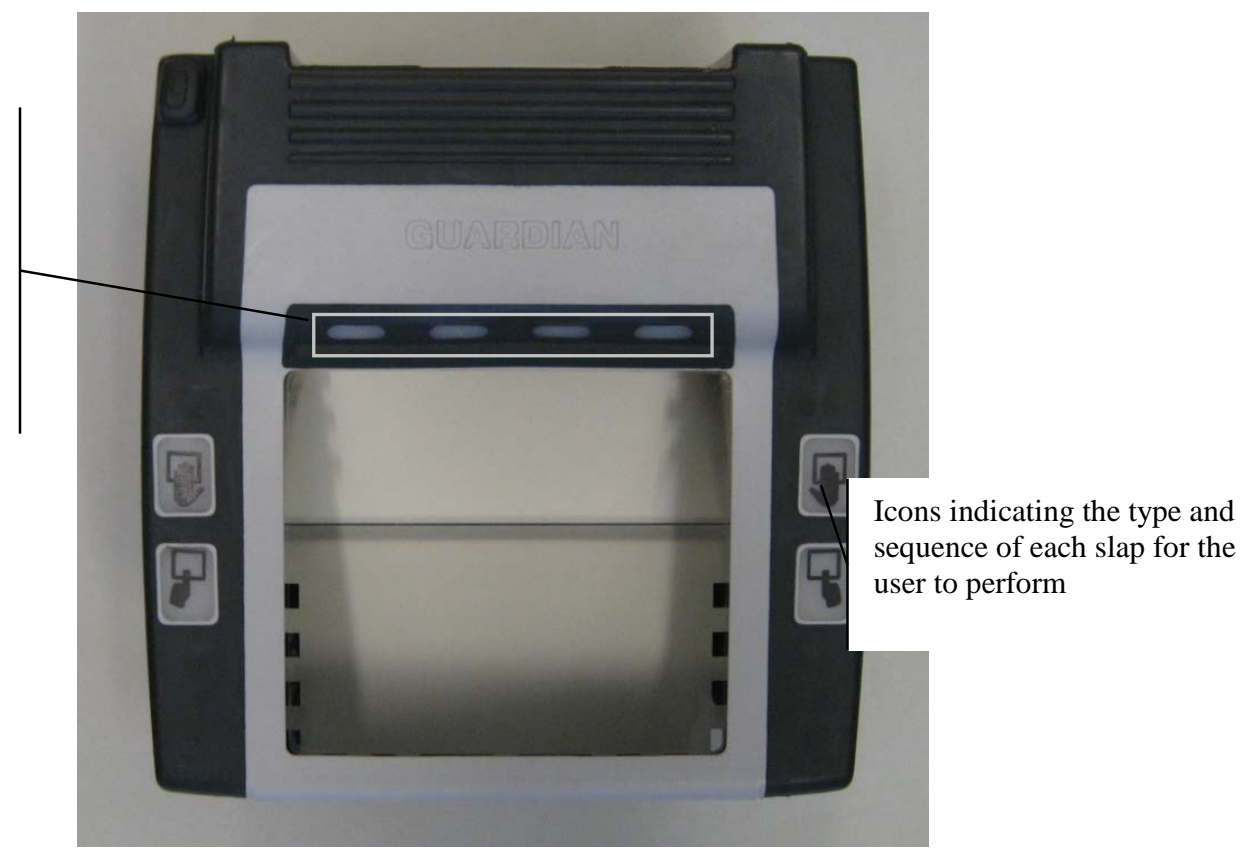

Figure 5: Fingerprint scanner with indicators

A custom capture application was used to control the fingerprint scanner and collect the digital images of participants' fingerprints. The LED lights, slap icons, and audible tones guided participants through the capture process.

When the scanner was ready, all of the LED lights above the platen were red and one of the four indicators on the sides of the platen was illuminated. The sequence in which the indicators were illuminated corresponded to the slap sequence: right four-finger slapand then left four-finger slap.

Once a participant placed fingers on the platen, the four LED lights indicated whether or not the scanner was able to read the user's fingerprints for the particular slap being requested.

When the user performed a right four-finger slap, the corresponding LED lights, e.g., the one on the far left for the index finger, the one on the far right for the little finger, turned from red to green if the scanner was able to read the appropriate fingerprints. Once all four lights turned green - indicating that all the fingers were readable - the scanner software checked the quality of the fingerprint image it was attempting to capture. After a few seconds, if the quality was acceptable, the scanner captured the image and beeped, indicating that it successfully captured an image. If the quality was insufficient, the scanner would stay on the same slap, until a successful capture and then moved to the next slap. 
The traditional scanner was placed on an adjustable table and angled at 20 degrees, resulting in a platen surface height of $91.44 \mathrm{~cm}$ (36 in), the recommended platen height (see Figure 7) [3].

\subsubsection{Instructional Materials}

Each of the scanners had an associated instructional video. The videos for the traditional contact scanner and the contactless scanner were developed by the researchers and depicted (with captioning) what hand to start with, the correct positioning of the fingers, and how to tell when the print had been accepted. The instructions were repeated for the other hand.

Before the test began each participant completed the demographic questionnaire shown in Appendix A. Once a participant filled out the demographic questionnaire, he or she was instructed to present his/her fingerprints three times to the traditional scanner and to one of the contactless scanners, each time under different conditions.

The fingerprint device the participants started with (contact or contactless) was counterbalanced across participants to control for learning effects - i.e., the first participant would use the traditional scanner first and the contactless scanner second for each task, while the second participant would use the scanners in the opposite order, and so on. Once a participant completed the fingerprinting tasks on the first device under the first two conditions they were asked to complete the tasks on the second device under the same two conditions.

Before interacting with the contactless scanner, the participants' hands were measured using the hand size estimator (see Figure 6) to initialize the software. All scanners were cleaned in between participants, when needed, to remove any visible fingerprints. 


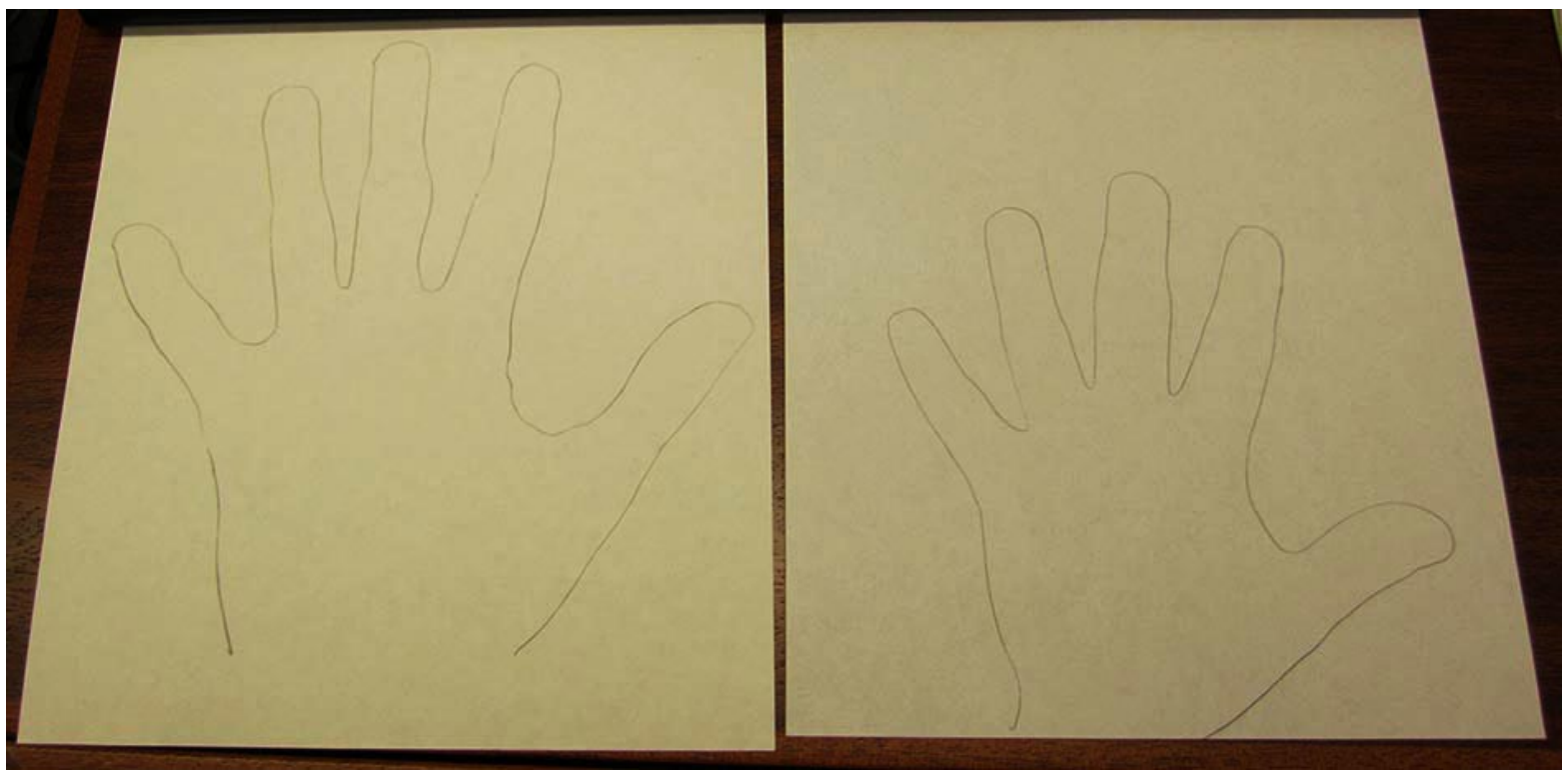

Figure 6: Hand Size Estimation Guides

For the first fingerprinting task (Task 1), participants were given no instructions whatsoever about how to use the scanners. Researchers verbally instructed participants to step up on to the mat (shown in Figure 7) "when you believe the device is ready to collect prints" and to step off "when you think the device has collected both sets of prints." 


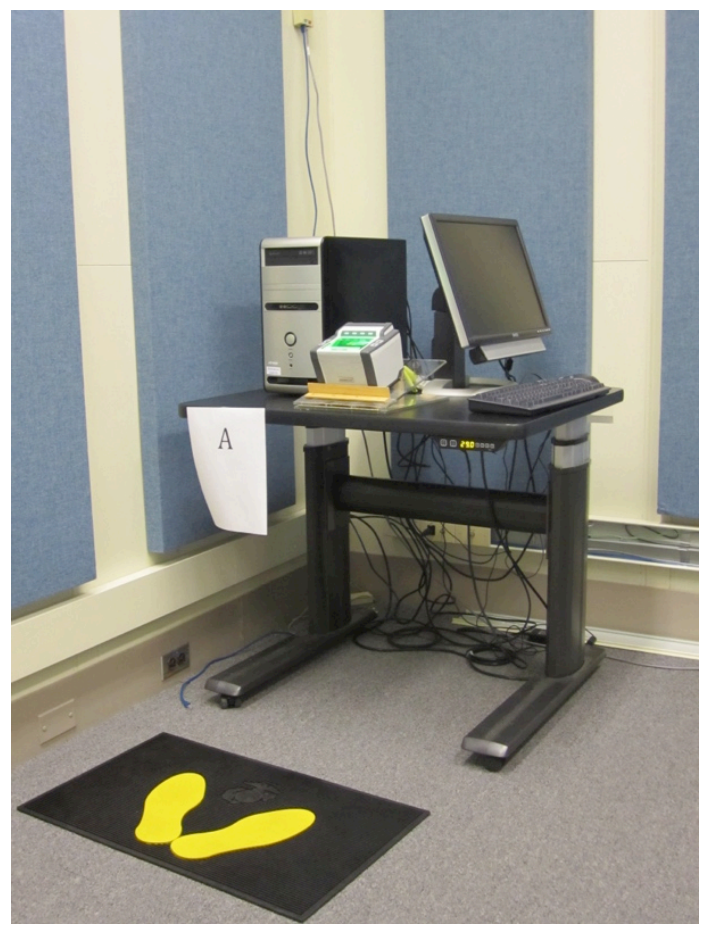

Figure 7: Fingerprint Scanner Setup Showing Mat and Adjustable Table

For the second task (Task 2), participants watched one of the instructional videos demonstrating the correct collection procedure for the scanner they were about two use. After viewing the video the participants were asked to complete the collection again, following the same verbal instructions used in Task 1.

After each participant completed both Task 1 and Task 2 on both scanners, he or she filled out the post-task questionnaire shown in Appendix B. Afterwards the participants were instructed to leave one more set of prints with each device they had used before (Task 3 ), this time with detailed verbal instructions on how to present prints for the device. These instructions were given before and, if needed, during the attempt.

\section{RESULTS}

This section describes the results of the usability tests performed on the contactless scanner, with comparisons to the results of tests on the traditional contact scanner used by all participants.

\subsection{USABILITY METRICS}

As described in the introduction to this document, this study was designed to test two contactless fingerprint scanner prototypes and measure their usability in terms of efficiency, 
effectiveness, and user satisfaction. These three dimensions of usability have specific definitions in the context of this study, described in the subsections below.

\subsubsection{Efficiency}

The efficiency of each device was measured in terms of how long it took for participants to complete fingerprinting tasks. Figure 8 details the testing timeline and sequence of activities for each task.

\subsubsection{Effectiveness}

The effectiveness of each fingerprinting device was measured in two ways: by task success and by the quality of captured fingerprints.

For purposes of this study, a successfully completed task was one in which the participant presented his or her fingers in such a way that the device was able to capture fingerprint images.

\subsubsection{User Satisfaction}

In this study, user satisfaction was measured by whether, and to what extent, participants found a given device intuitive, easy to use, fast, and good at providing feedback. User satisfaction data was collected through the use of the post-task questionnaire in Appendix B.

There were five questions to which participants responded with a binary (e.g., yes/no) answer and comments, as well as two questions to which they responded with comments only. Participants provided two sets of responses per question, one set for the contact scanner and one set for the contactless scanner. 


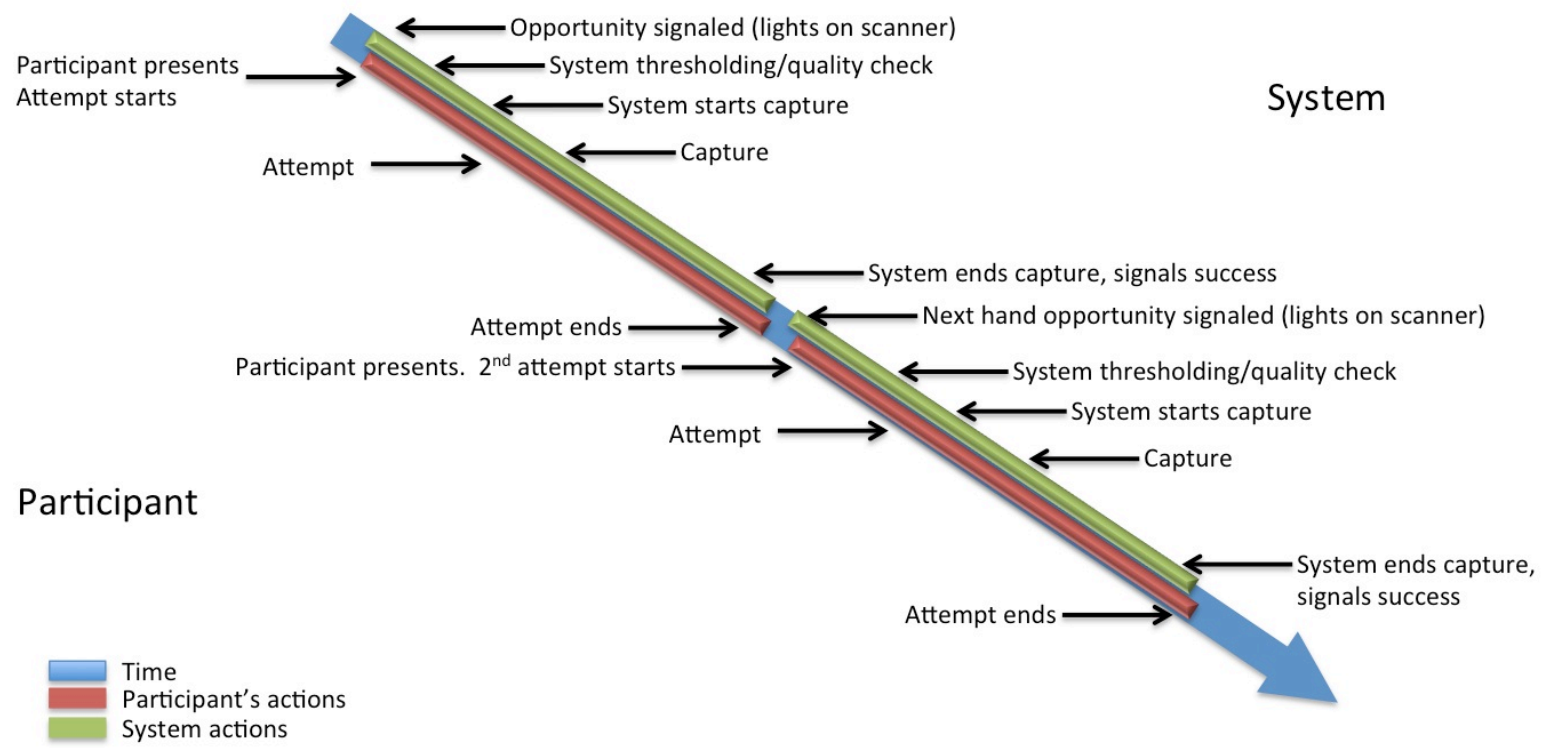

Figure 8: Testing Timeline 


\subsection{CONTACTLESS SCANNER}

\subsubsection{Efficiency}

The average amount of time it took for participants to complete fingerprinting tasks with the first contactless scanner and the contact scanner are shown in Table 1 below.

Table 1: Average Task Times in Seconds - Contactless Scanner

\section{Time to Complete Task (in seconds)}

\begin{tabular}{l|c|c|c} 
& $\begin{array}{c}\text { Task 1 (no } \\
\text { instructions) }\end{array}$ & Task 2 (video) & $\begin{array}{c}\text { Task 3 (verbal } \\
\text { instructions) }\end{array}$ \\
\hline Contact Scanner & 47.77 & 35.08 & 21.61 \\
\hline Contactless Scanner & 80.05 & 118.39 & 72.85
\end{tabular}

As the table indicates, it took longer, on average, for the contactless scanner to capture participants' fingerprints than it did for the contact scanner to do so.

\subsubsection{Effectiveness}

Table 2 shows the number of participants who had their prints collected by each device in Task 1, Task 2, and Task 3. The rate of success was higher for the contact scanner than for the contactless scanner.

Table 2: Successful Print Collection per Task - Contactless Scanner

\# of participants (out of 61) who successfully completed the fingerprinting task

\begin{tabular}{l|c|c|c} 
& $\begin{array}{c}\text { Task 1 (no } \\
\text { instructions) }\end{array}$ & Task 2 (video) & $\begin{array}{c}\text { Task 3 (verbal } \\
\text { instructions) }\end{array}$ \\
\hline Contact Scanner & 38 & 55 & 60 \\
\hline Contactless Scanner & 11 & 34 & 58
\end{tabular}




\subsubsection{I mage Quality and Suitability for Matching}

There are several approaches that can be utilized to measure the suitability or effectiveness of the captured fingerprint images for the intended task of biometric identification or verification. These approaches include subjective analysis of images by a trained human observer, monolithic means of quality measurement by an algorithmic process, and conducting an verification or identification classification using an actual matcher. Each approach has benefits and drawbacks that will be discussed in their respective sections.

\subsubsection{Assumptions and Exclusions}

Of the 61 participants a subset of 51 were down-selected. The reason for this downselection of data is because some of the participants failed to leave the expected set of 8 fingerprints on either contactless scanner, contact control scanner, or both. In other cases, images from the contactless scanner failed to generate a rolled-equivalent representation of the captured finger in post-processing. The down-selected set of 51 participant provides complete data for every participant where both the contactless as well as contact control scanners successfully acquired and captured all available fingers, and the contactless scanner solution generated a rolled-equivalent image representation of the contactless capture. For matcher testing (only), a subset of 50 of the 51 participant was down-selected and one participant's data was rejected. The reason for the rejection of one participant was due to a data capture error during collection causing an identity mismatch for one participant between the contactless scanner captured data and the control contact control scanner.

\subsubsection{Observed I mage Anomalies}

The effectiveness of human observation of image quality for the purpose of predicting the success of automated matching in identification or verification scenarios has been a much debated topic. In one camp, the examiner observation of image quality is taken as the gold standard in quality measurement while in another camp the question of quality is presented as something that can only be answered by machine-based matching regardless of what a human observer deems as a visually optimal fingerprint.

For the purpose of this study a less exhaustive observer-based examination of images was conducted in favor of also conducting a matcher based examination of image quality. The observations conducted for this study were primarily to detect overt anomalies in the contactless images as a result of capture or post-processing and in no way attempt to quantify 
anomalies related to the intrinsic quality of the fingerprint. Where needed, the contact-based images were used as a reference to validate that the anomalies are indeed related to the capture process and not due to any intrinsic features of the finger.

Various image defects were noted. Figure 9 through Figure 13 illustrate examples of information loss from one or more image planes or other image defects likely to compromise utility of the images as rolled equivalent fingerprints. Extreme information loss is illustrated by Figure 17 appearing later in this section.

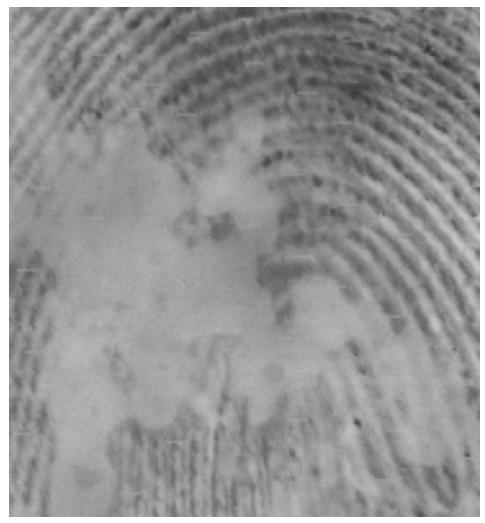

Figure 9 Blotchy information loss.

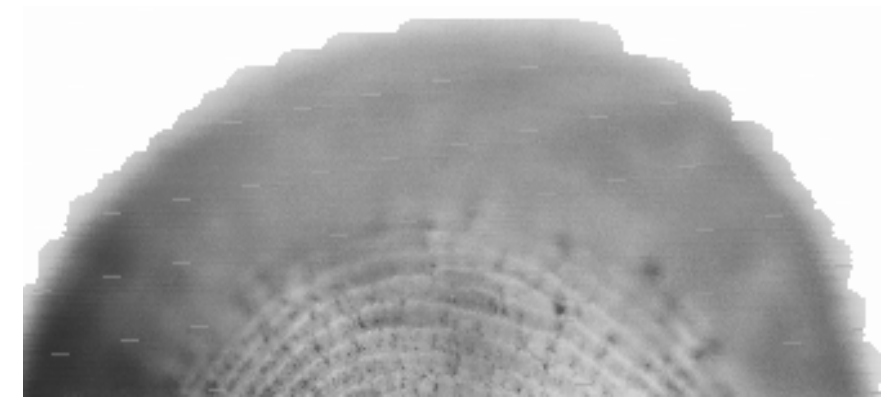

Figure 10 Image plane fusion artifact/visible layering boundaries. 


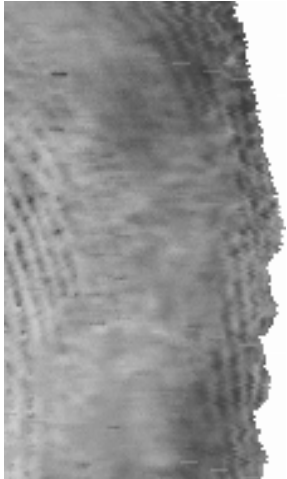

Figure 12 Image plane fusion blurriness/loss of signal energy in one plane and Zigzag/harmonic distortion pattern on edges of image

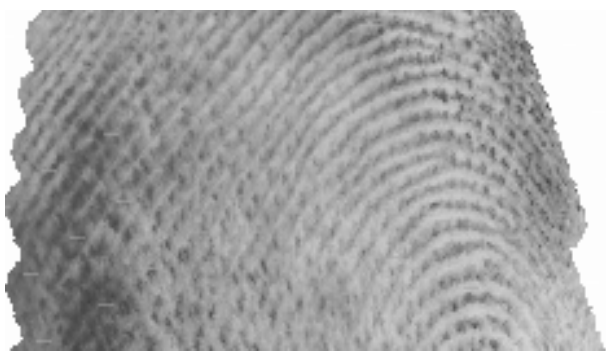

Figure 13 Oblique view

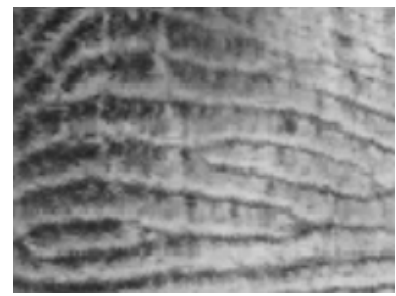

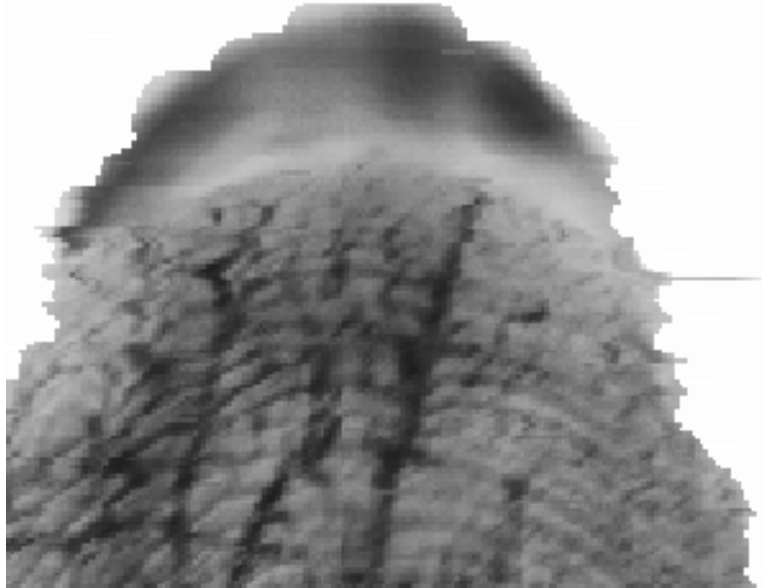

Figure 11 Fingernail imaged (top-most capture plane not rejected) and Image tearing.

Figure 14 Demonstration of contrast reversal of contactless scanner

- note that dark broad ridges of delta in image on left grade into light tones as valleys grade from light to dark. Image on left is corresponding print from contact scanner provided for comparison. 


\subsubsection{Monolithic Quality Measures}

While it is widely accepted that the best measure of the suitability of a fingerprint image for the purposes of identification or verification is to actually conduct a match, many times it is not possible to do so for various reasons such as not having a fingerprint repository to do so at the time of capture. In these cases, an image has been captured and a predictor of suitability for processing that image later through a matcher [at a later time] needs to be calculated using the single image on hand. The image can be subjected to any number of signal processing techniques or algorithms to classify or quantify the suitability of the image for the purposes of matching. This is referred to in this study as a monolithic quality measure and several such measures are explored in this study.

\subsubsection{Signal Analysis Based Measures}

The images from the contactless scanner were observed to be positioned with the long axis horizontal rather than vertical as is the conventional presentation of fingerprints.

Individual "flat" images were segmented from the four-finger slap impressions acquired with the Contact Control Scanner.

Subjects for which a complete complement of eight finger impressions were not present for both scanners were eliminated from the analysis. This screening yielded eight prints for each of 51 subjects or $\mathrm{N}=408$ fingerprint samples for each of the two scanners.

\subsection{Image Entropy}


Image entropy is a measure of the use of the available gray scale of the 8-bit image, i.e. the degree to which the histogram having bins 0 to 255 is populated. It is defined as

$$
E=-\sum_{i=0}^{255} p\left(x_{i}\right) \log _{2} p\left(x_{i}\right)
$$

where $p\left(x_{i}\right)$ is the probability (proportion) of image pixels having gray level $x_{i}, i=0 \ldots 255$.

Inspection of the boxplot [6] of Figure 15 shows a fairly tight distribution of entropy values for the contactless scanner about a median of 6.0 bits. The Contact Control Scanner shows lower entropy values distributed about a median of 3.8 bits. 


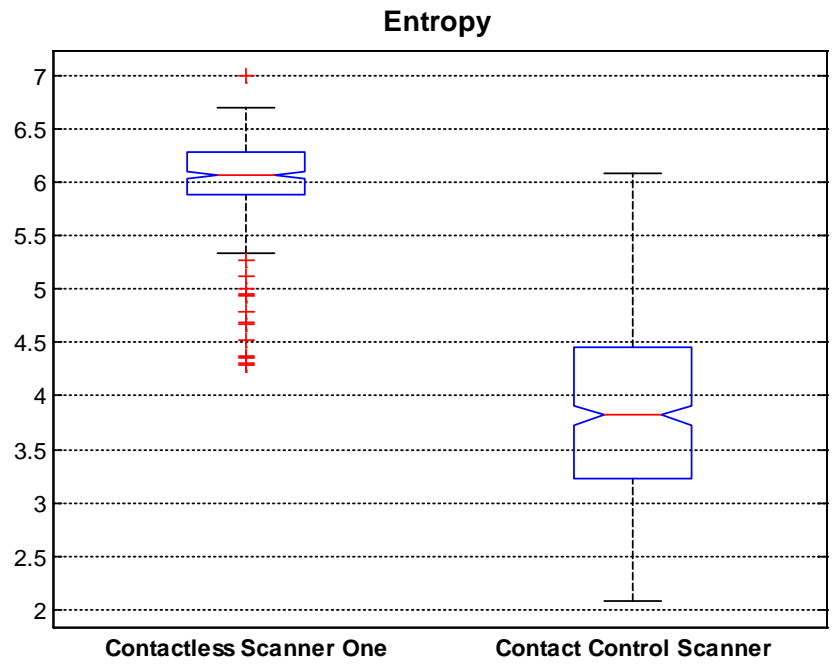

Figure 15 Distribution of entropy values for Contactless Scanner compared with entropy distribution of Contact Control Scanner.

Image entropy can be spuriously lowered by large areas of uniform gray level such as white space surrounding the fingerprint impression. A few examples fingerprints from each scanner were cropped tightly from their surrounding white space backgrounds. The entropy for the Contact Control Scanner increased to approximately 5.5 to 6.0 bits. Similar cropping of the contactless scanner impressions yield entropy values approaching 7.0 bits. With such cropping, both scanners show improvements in use of the available grayscale, with the contactless scanner images approaching entropy values observed with inked fingerprints. However, the observation of contrast reversals between ridges and valleys (see Figure 14) and effects of uneven illumination observed with the contactless scanner somewhat diminishes the value of entropy gains.

\subsection{Blind Signal to Noise Ratio (BSNR)}

The blind signal to noise ratio (BSNR) is essentially a measure of the sharpness of the image as it is a function of the edge energy of an image that exceeds a threshold. In this respect a low contrast or blurred image will show less edge energy than a high contrast, sharply focused image. We employ an algorithm adapted from Zhang and Blum [7]. 
Given an image, $I$, we compute the directional gradients at each image location as

$$
\begin{aligned}
& \frac{\partial I(x, y)}{\partial x}=\frac{I(x+1, y)-\mathrm{I}(x-1, y)}{2} \\
& \frac{\partial I(x, y)}{\partial y}=\frac{I(x, y+1)-\mathrm{I}(x, y-1)}{2}
\end{aligned}
$$

We then compute the Euclidean norm of the gradient as

$$
\mathbf{G}_{x, y}=\left[\left(\frac{\partial \mathbf{I}_{x, y}}{\partial x}\right)^{2}+\left(\frac{\partial \mathbf{I}_{x, y}}{\partial y}\right)^{2}\right]^{\frac{1}{2}}
$$

We set a threshold by which to select those values of $\mathbf{G}_{x, y}$ such that

$$
\mathbf{G}_{x, y} \geq 2\left(\frac{\sum_{x=1}^{N} \sum_{y=1}^{M} \mathbf{G}_{x, y}}{N M}\right)=2 \mu_{\mathbf{G}}
$$

we can define a set of pixels, s, meeting the threshold condition, i.e.,

$$
s=\left\{\mathbf{G}_{x, y} \mid \mathbf{G}_{x, y} \geq 2 \mu_{\mathbf{G}}\right\}
$$

The proportion of image pixels having gradient exceeding the threshold is then

$$
q=\frac{|s|}{N M}
$$

where the $|s|$ in this case denotes the number of elements in satisfying the threshold criterion.

The blind signal to noise ratio is then defined as 


$$
B S N R=20 \log \left(\frac{q}{e^{-\pi}}\right)
$$

Inspection of the boxplot shown in Figure 16 shows both higher signal to noise ratio for the Contact Control Scanner and much less variability with fewer outliers.

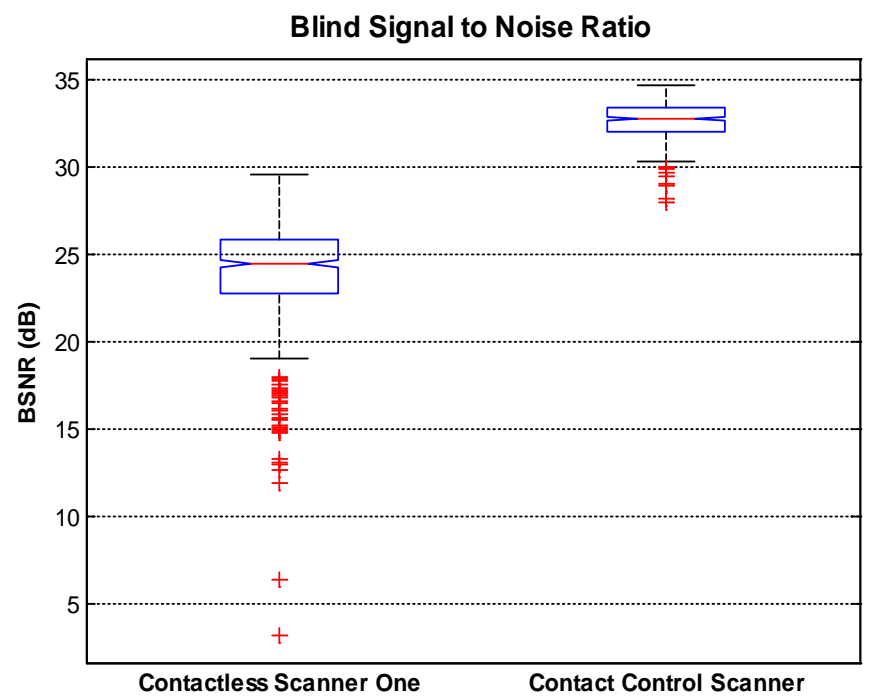

Figure 16 Distributions of blind signal to noise values for Contactless Scanner and Contact Control Scanner.

To provide insight into a lower bound for the BSNR metric relative to these fingerprint data, we compute the median of approximately $17 \mathrm{~dB}$ for fingerprint images judged subjectively to have lost all or close to all ridge detail such as that shown in Figure 17. Several images showing even greater blurring that that shown in Figure 17 had BSNR values as low as $3 \mathrm{~dB}$. Thus, edge feature definition is significantly higher with the Contact Control Scanner than that of images acquired with the contactless scanner. Moreover, the apparent loss of edge energy in many of the images is related to poor focus or loss of detail from some to all image planes used in construction of the rolled equivalent fingerprint. It is unlikely that the photographic process used with the contactless scanner can approach the edge energy observed with the FTIR contact device, as edge energy will normally be lower in higher entropy images as found in our informal testing on a variety of fingerprint image types. The 
greater difficulty for the contactless scanner is the extension of the distribution toward the lower extremes. 


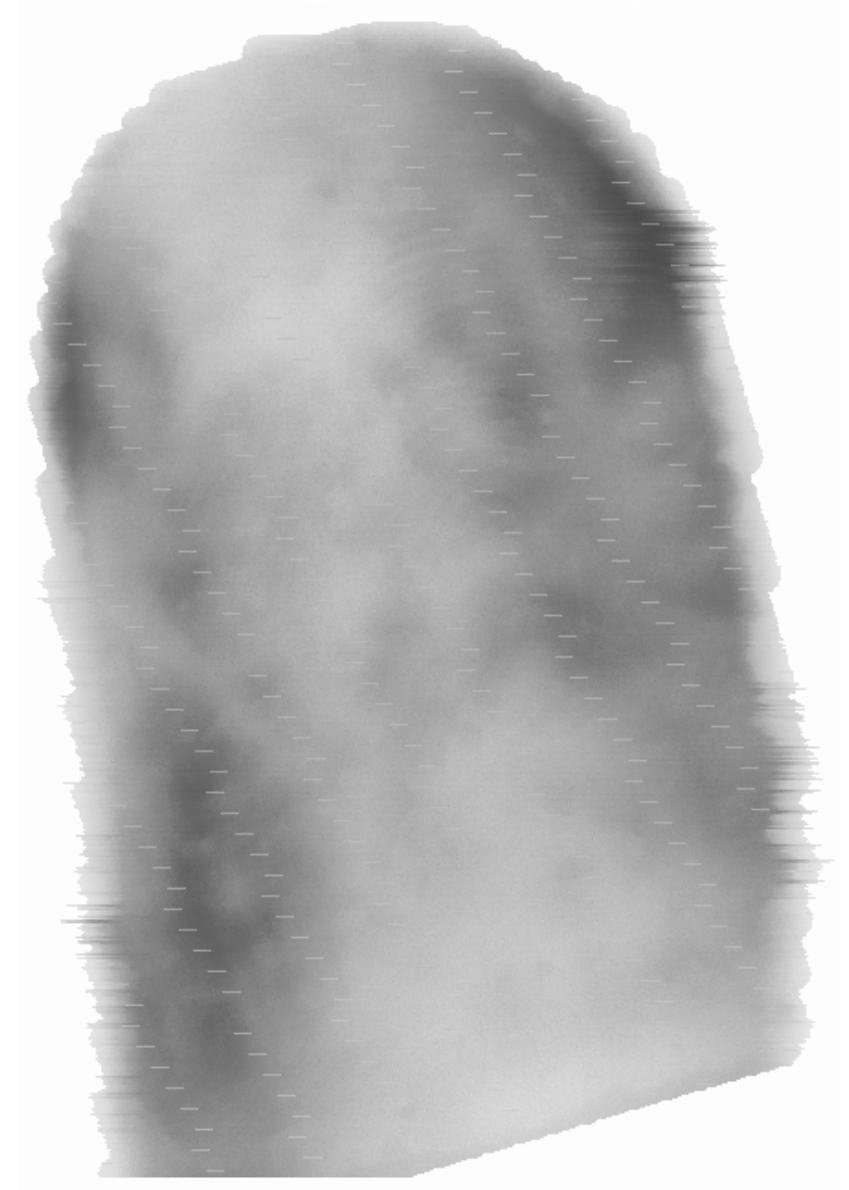

Figure 17 Example of complete loss of ridge detail observed with an image acquired with Contactless Scanner illustrating low BSNR value of approximately 15. 


\subsubsection{NIST Spectral I mage Validation/ Verification (SI V) Measures}

The NIST Spectral Image Validation/Verification (SIVV) metric [8] was developed to provide a simplified representation of the frequency spectrum of fingerprint images. Several properties of the SIVV signal provide some insight as to the strength of the fingerprint ridge pattern, and clues as to whether observed ridge spacing is that expected of a fingerprint acquired at a particular sample rate.

Frequency location of the largest relative peak of the signal provides a measure suggestive of the ridge spacing of the fingerprint image respective of the sample rate at which the image was acquired. Our objective in evaluating the frequency location of the peaks is to ascertain that the location is approximately at $0.1-0.15$ cycles/pixel, the appropriate range for $500 \mathrm{ppi}$ images, and to assess the dispersion of the distribution of peak locations as a measure of the strength of the ridge frequency relative to artifact patterns at various frequencies. Figure 18 shows that the median peak locations for both scanners are similarly located, confirming a 500 ppi sample rate for both image types. However, the dispersion is much greater for the contactless scanner that together with the large number of outliers shown in the boxplot indicates less consistency in expression of the ridge pattern for the contactless scanner fingerprints. In this regard we note that a fingerprint image lacking ridge detail will fail to show a peak where expected in the SIVV signal. The SIVV algorithm in this case will select the largest peak from elsewhere in the spectrum, often in the high frequency. Indeed, as pointed out in the subjective visual assessment of the images, many are decidedly blurred or have small to large areas of the ridge structure masked by blurred patches. 


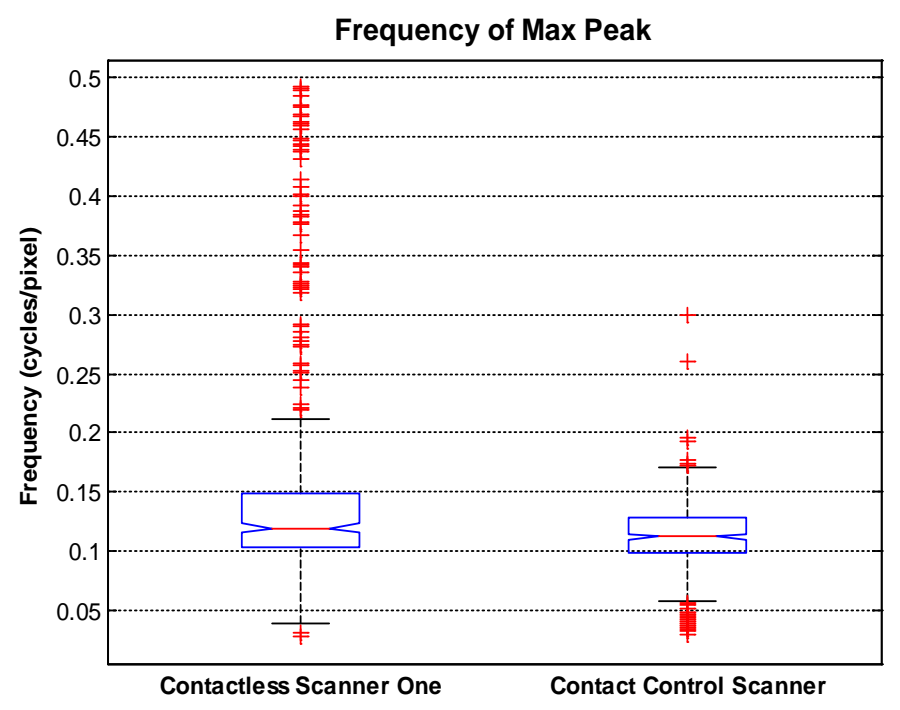

Figure 18 Center frequency of maximum SIVV signal peaks for Contactless Scanner and contact control scanner.

As shown in Figure 19, the height of peaks tend to be smaller for the contactless scanner compared to the Contact Control Scanner suggesting a stronger ridge expression with this device in comparison to the contactless device. This could result from the lower local contrast images of the contactless scanner and less pronounced ridge pattern than that of the Contact Control Scanner. 


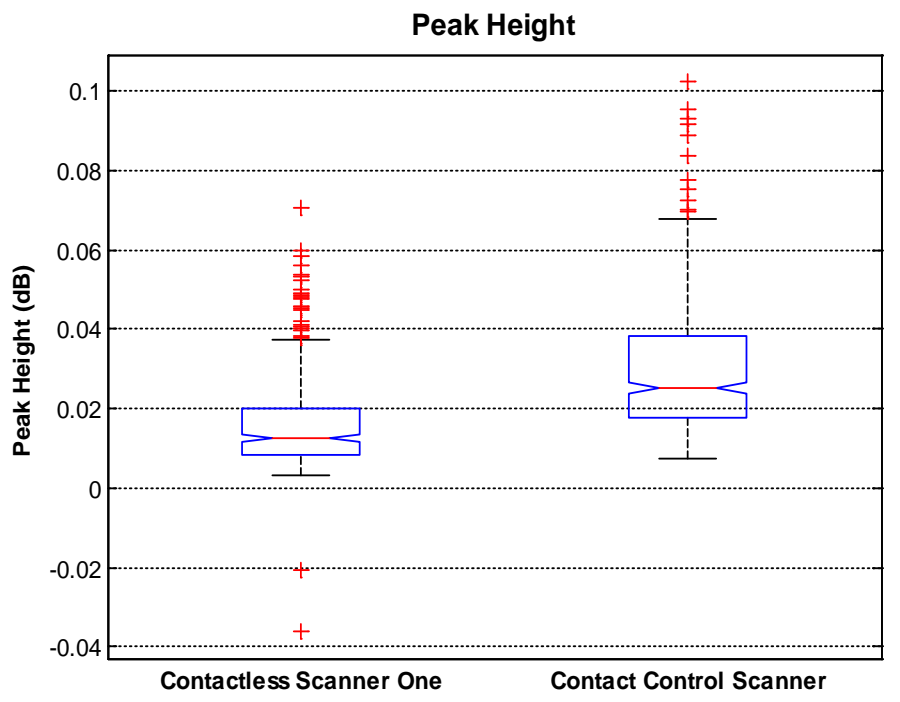

Figure 19 Relative height of SIVV signal peaks for Contactless Scanner and contact control scanner. 


\subsubsection{Conclusions - Monolithic Quality Measures}

The spectral analysis result verifies that the contactless scanner appears to sample the friction ridge pattern at the expected $500 \mathrm{ppi}$, though a number of image reconstruction anomalies increase the variance of both the position and power of the spectral peak corresponding to the ridge pattern. The contactless scanner images exhibit signal to noise characteristics (BSNR) on the order of those observed with databases of inked rolled fingerprint impressions, but due to lower contrast with attendant lower edge energy exhibit BSNR lower than that of fingerprints acquired with the Contact Control Scanner.

Though comparable in a statistical sense to a random sample of inked rolled fingerprints with regard to BSNR (a measure related to contrast), the major problem with the contactless scanner is that contrast frequently reverses between ridge and inter-ridge across the image surface. Thus, algorithms attempting to follow ridges or detect features must somehow reconcile the change in the dark-to-light relationship. While the human eye is well adapted to accommodate such transitions, the machine may not be able to adjust to such dynamics.

Moreover, a number of problems are observed regarding assembling the images from the planar samples that either distort, or obscure features necessary for fingerprint identity matching. In many cases loss of information around the periphery of the print renders it ineffective as a "rolled equivalent" even if it would suffice as a flat. Where the loss of information occurs near the region of the fingerprint core, the entire print becomes unusable. In other cases, horizontal "tearing' of the image distorts ridge features from their actual path and can even introduce intersections or endings to ridges that do not actually exist. As a related issue, virtually every image contains a repetitive pattern of lines orthogonal to the long axis of the finger that may appear as false minutiae to a machine algorithm.

\subsubsection{NFIQ Based Measure}

The NIST Fingerprint Image Quality algorithm (NFIQ) [2] presents a more robust method of predicting the suitability of an image for the purposes of automated matching. NFIQ relies on both signal based metrics of image quality as well as machine learning techniques to classify the quality of a single image for the purpose of predicting matchability in an identification or verification setting. The classifications provided by NFIQ can range from 1 


\begin{tabular}{|c|c|c|c|c|c|c|c|c|c|c|c|c|c|c|c|c|c|}
\hline & & \multicolumn{8}{|c|}{ Contactless Scanner } & \multicolumn{8}{|c|}{ Contact Control Scanner } \\
\hline $\begin{array}{l}\text { NFIQ } \\
\text { Classificati } \\
\text { on }\end{array}$ & 㺃 & 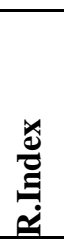 & 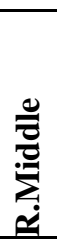 & في & 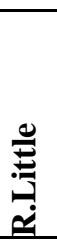 & تِ & 莺 & 苞 & 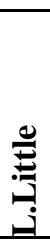 & 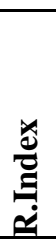 & 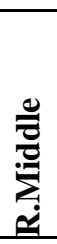 & بـ & 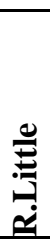 & & 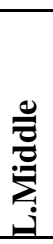 & : & 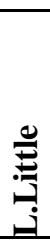 \\
\hline & 1 & 1 & 0 & 2 & 2 & 0 & 1 & 1 & 0 & 19 & 21 & 16 & 6 & 18 & 22 & 17 & 10 \\
\hline & 2 & 0 & 0 & 1 & 0 & 0 & 0 & 0 & 0 & 11 & 6 & 5 & 8 & 6 & 3 & 7 & 5 \\
\hline & 3 & 8 & 11 & 7 & 5 & 11 & 8 & 6 & 7 & 13 & 11 & 20 & 15 & 18 & 16 & 10 & 15 \\
\hline & 4 & 19 & 17 & 16 & 19 & 21 & 18 & 17 & 7 & 3 & 6 & 6 & 3 & 3 & 4 & 9 & 5 \\
\hline & 5 & 22 & 22 & 24 & 24 & 18 & 23 & 26 & 36 & 4 & 6 & 3 & 18 & 5 & 5 & 7 & 15 \\
\hline
\end{tabular}

(high probability of suitability) to 5 (low probability of suitability). The classifications provided by NFIQ are discrete and do not represent a continuous function of quality measurement.

Images from both the contactless scanner and the Contact Control Scanner were passed through NFIQ and summary results are provided in the Table 3 and the boxplot presented in Figure 20.

Table 3 - Count of NFIQ scores for Contactless Scanner and Contact Control Scanner 


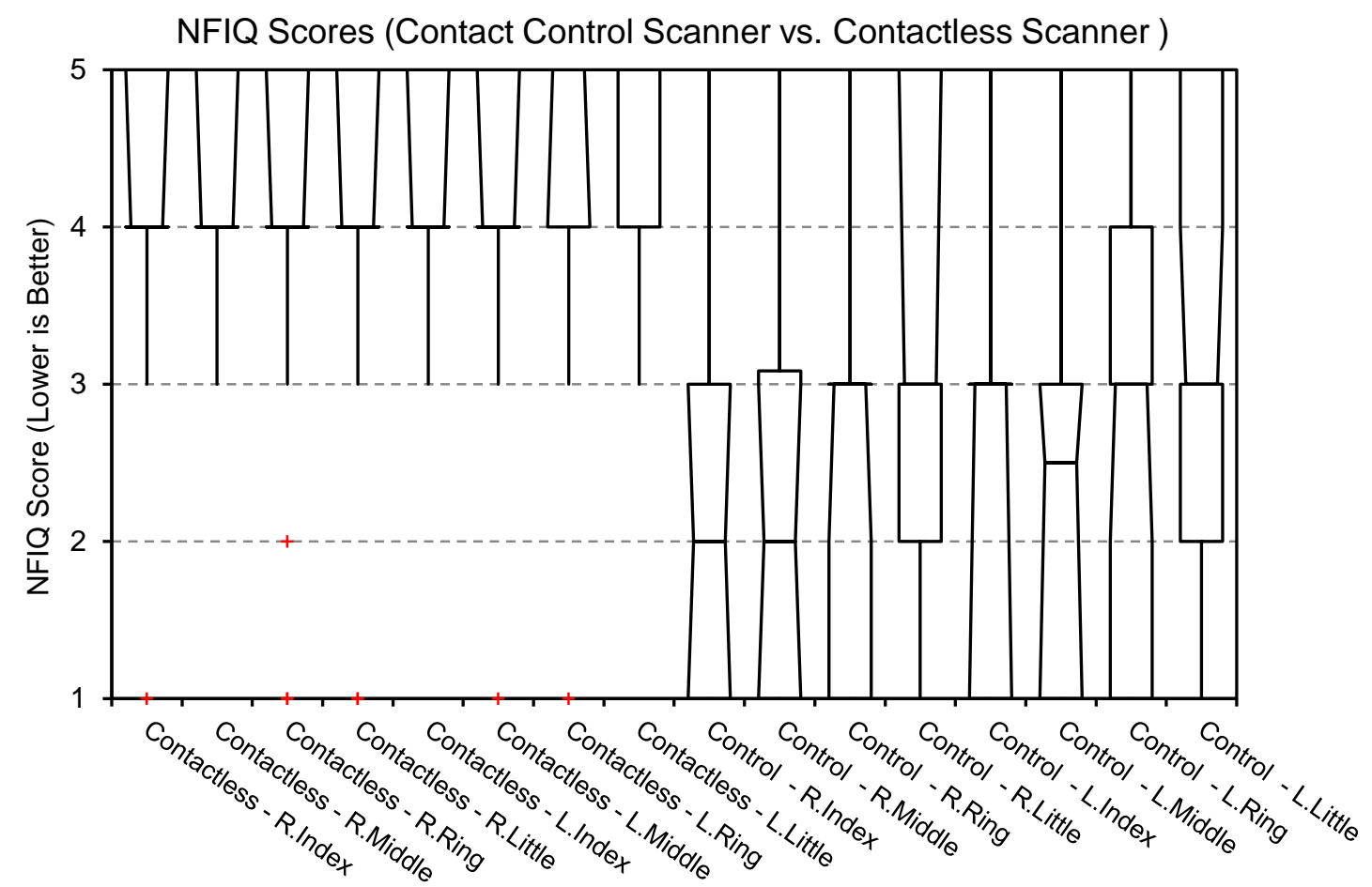

Figure 20 - Boxplot of NFIQ Scores (Contactless Scanner vs. Contact Control Scanner)

Cursory examination of the boxplot (Figure 20) shows that scores from the contactless scanner are consistent clustered around lower quality levels $(4,5)$ while scores from the Contact Control Scanner are consistently clustered at higher quality classifications $(1,2,3)$.

This observation was confirmed via pairwise comparison of NFIQ from each finger between the contactless scanner and the Contact Control Scanner. Wilcoxon Signed Rank Test [9][10] showed that this difference was statistically significant below the 0.05 level for all cases (Table 4). 
Table 4 - Statistical Comparison of NFIQ scores from Contactless Scanner and Contact Control Scanner.

\begin{tabular}{|c|c|c|c|c|c|c|c|c|}
\hline & 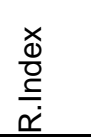 & 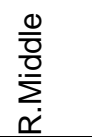 & 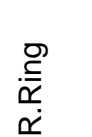 & 岇 & 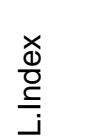 & $\frac{\stackrel{0}{\frac{0}{0}}}{\sum_{j}^{\frac{0}{2}}}$ & 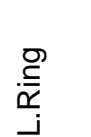 & $\stackrel{\varrho}{\stackrel{D}{E}}$ \\
\hline $\begin{array}{r}\text { 3.2.3.1.1 p, Wilcoxon two-tailed test } \\
\mathrm{R} 1<>\mathrm{R} 2\end{array}$ & $\begin{array}{c}<0.000 \\
1\end{array}$ & $\begin{array}{c}<0.000 \\
1\end{array}$ & $\begin{array}{c}<0.000 \\
1\end{array}$ & 0.0002 & $\begin{array}{c}<0.000 \\
1\end{array}$ & $\begin{array}{c}<0.000 \\
1\end{array}$ & $\begin{array}{c}<0.000 \\
1\end{array}$ & $\begin{array}{c}<0.000 \\
1\end{array}$ \\
\hline \multirow[t]{3}{*}{ NFIQ Favors Contact Control Scanner (n) } & 43 & 41 & 45 & 30 & 41 & 44 & 32 & 32 \\
\hline & 1 & 0 & 2 & 6 & 1 & 1 & 2 & 2 \\
\hline & 6 & 9 & 3 & 14 & 8 & 5 & 9 & 16 \\
\hline
\end{tabular}

\subsubsection{Matcher Based Quality Metrics}

For machine matcher-based quality metrics, two research applications from the NIST NBIS (NIST Biometric Image Software) distribution v 4.1.0 were utilized:

1. MINDTCT - The NIST Minutiae Detector application generates a minutiae template according to the ANSI INCITS 378-2004 standard from a source fingerprint image

2. BOZORTH3 - The NIST Bozorth-3 matcher application generates a match score from an input consisting of two fingerprint minutiae templates

All images captured by the contactless scanner as well as the Contact Control scanner were processed by MINDTCT to obtain minutiae templates, and the resulting templates were then matched by the BOZORTH3 matcher to obtain match scores for pairs of templates.

In examining the suitability of fingerprints captured from the contactless scanner for identification purposes we modeled three identification scenarios:

1. Contactless Scanner to Self: This case models the operation scenario where fingerprints from the contactless scanner are compared to a gallery of other images 
captured by the same scanner without any legacy operational contact-based data (i.e., new to new).

2. Contact Control Scanner to Self: This case models the operational scenario of contact-based scanners generating images for comparison against a gallery of contactbased scanner images (i.e., legacy to legacy).

3. Contactless Scanner to Contact Control Scanner: This case models the operational scenario where fingerprints from the contactless scanner are compared to a gallery of images from a contact-based scanner (i.e., new to legacy interoperability).

For each of the three identification scenarios above, we generated score distributions for two cases, mated-identification (ident) and non-mated identification (non-ident) scenarios. To generate the mated-identification scores, the same fingerprint template from a given participant was matched to itself providing an expected ident with an optimal match score. ${ }^{2}$ For the non-mated identification cases each participant's fingerprints were matched against the next available participant in the data set (for example, participant 1 was matched against participant 2, participant 2 to 3, and so on).

Once mate and non-mate scores were obtained for each of the three identification scenarios, the three scenarios were compared to each other by calculating performance characteristics. These performance characteristics include FMR (False Match Rate, or the rate that impostors are incorrectly identified as genuine mates) and FNMR (False Non Match Rate, or the rate that genuine mates are incorrectly identified as imposters). Calculations were performed by setting an operational target FMR of 5\% and selecting a match threshold (integer) that would come closest to this target.

Comparison of the mate and non-mate scores from the Contact Control Scanner to itself using a matching threshold of 16 shows that FMR is at approximately $5.5 \%$ while FNMR is zero. Matching images from the contactless scanner to other images from the contactless scanner yield an FMR of 4.5\% and an FNMR of 9.0\%. This finding is rather disturbing in that matching of images to themselves in this context is a rather trivial challenge. Further examination of the failure of self-matches for the contactless scanner indicated a sizeable number of fingerprints captured by this device generated fingerprint templates with too few

\footnotetext{
2 This "self-ident" approach was used in lieu of no suitable mated impressions being available from the test collection protocol.
} 
(or no minutiae). A cursory evaluation of a subset of these images shows that some of the images simply lack any ridge detail detectable to the human eye.

A cross-comparison of images captured by the Contact Control Scanner matched against images captured by the contactless scanner with a target FMR of 5\% yields an FMR of $4.8 \%$ at a matcher threshold of 14, and an FNMR of 70.0\%.

Table 5 - Match Performance Summary for Identification Scenarios

\begin{tabular}{|clccc|}
\hline Identification Scenario & FMR & FNMR & Match Threshold \\
\hline 3.2.4.1.1 & $\begin{array}{l}\text { Contact Control Scanner } \\
\text { to Self }\end{array}$ & $5.5 \%$ & $0.0 \%$ & 16 \\
\hline 3.2.4.1.2 & $\begin{array}{l}\text { Contactless Scanner to } \\
\text { Self }\end{array}$ & $4.5 \%$ & $9.0 \%$ & 16 \\
\hline 3.2.4.1.3 & $\begin{array}{l}\text { Contactless Scanner to } \\
\text { Control }\end{array}$ & $4.8 \%$ & $70.0 \%$ & 14 \\
\hline
\end{tabular}



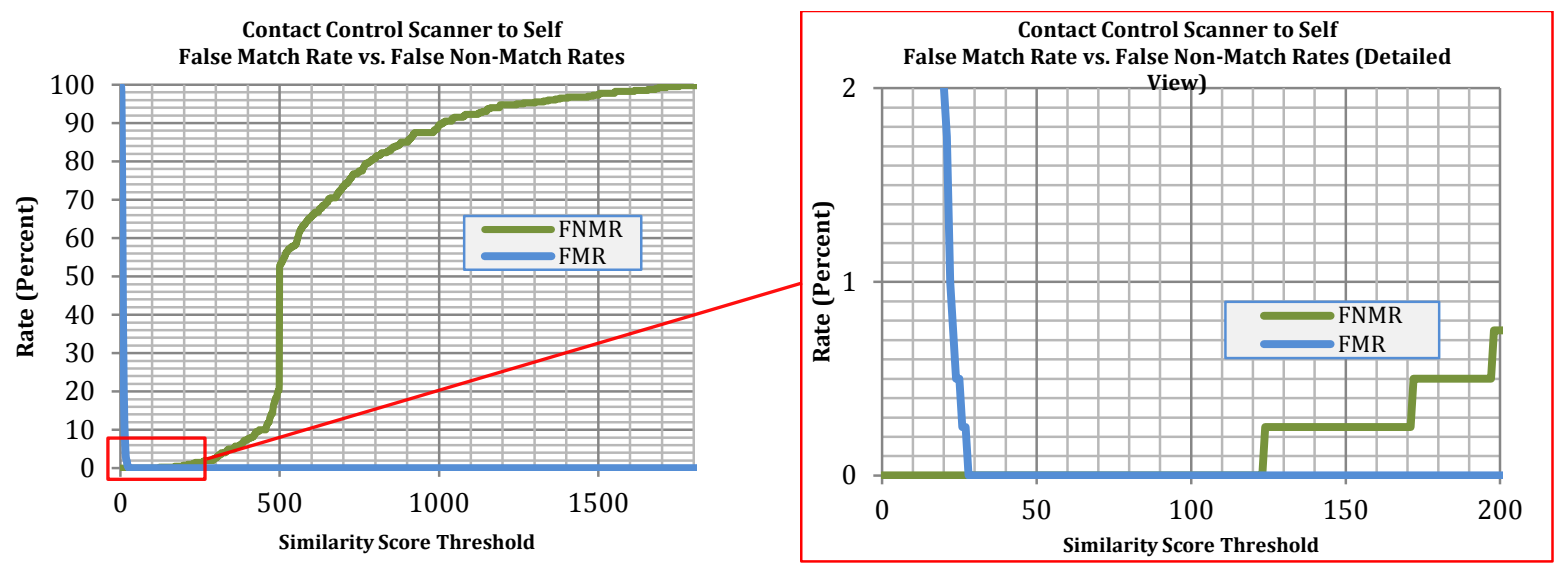

Figure 21 - Cumulative FMR/FNMR Probability graph for Contact Control Scanner Matches to Self
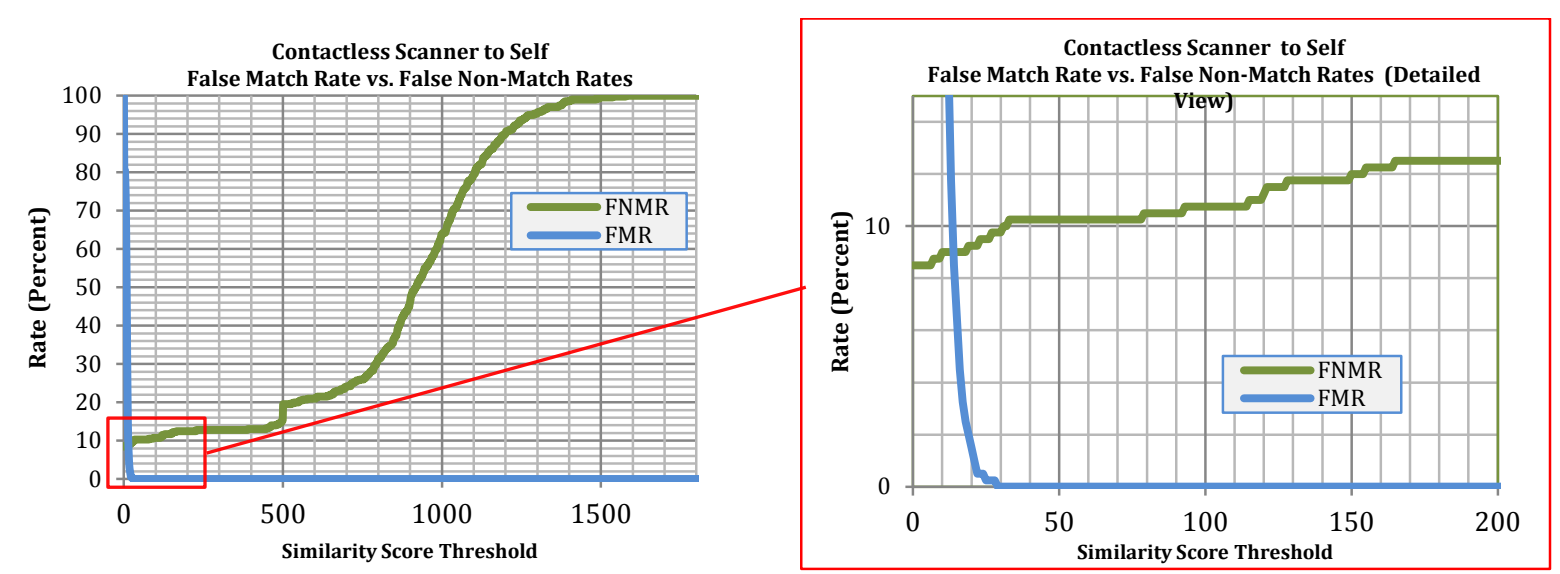

Figure 22 - Cumulative FMR/FNMR Probability graph for Contactless Scanner Matches to Self 

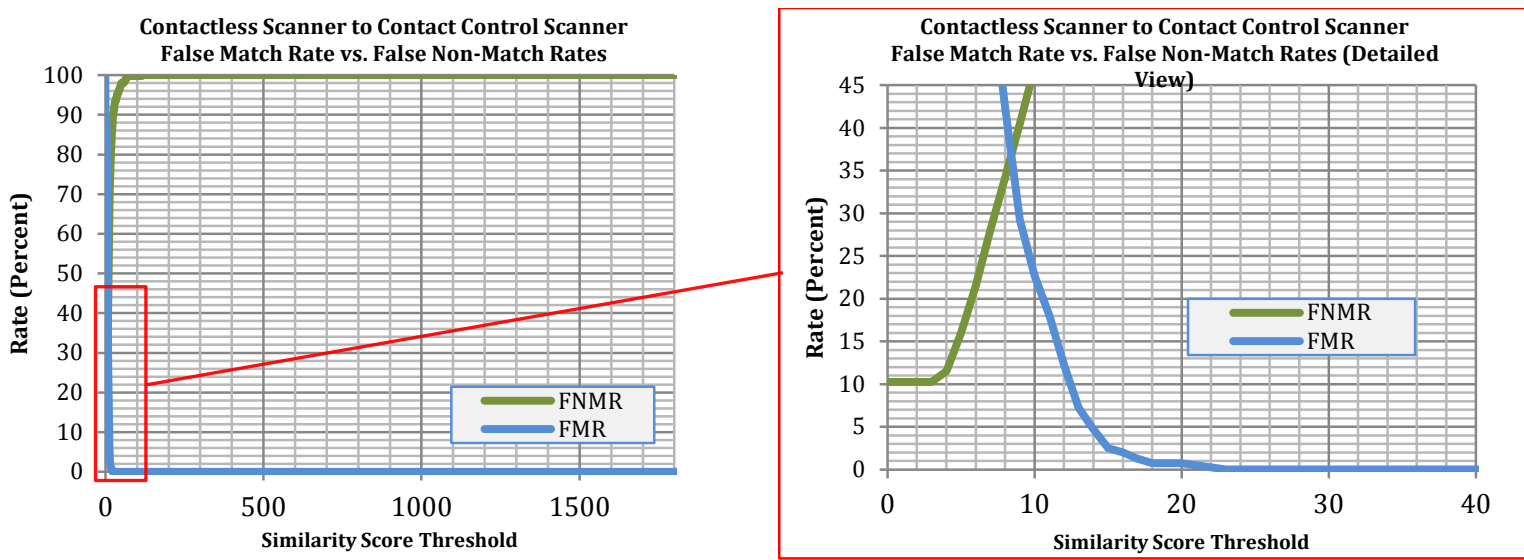

Figure 23 - Cumulative FMR/FNMR Probability graph for Contact Control Scanner Matches to Contactless Scanner 


\subsubsection{Summary of Findings}

Testing of images captured by the contactless scanner showed that images from this scanner can be processed using the various tools normally used to assess the quality of digital fingerprint imagery captured using traditional contact-based scanners. While processing with these tools in no way guarantees that the tools behave the same on images derived from contactless scanners in the same way that they with legacy contact-based images, it was shown that these images can be assessed for quality, processed to detect minutiae and generate minutiae matching templates, and the resulting minutiae templates matched against other fingerprint minutiae templates.

Computational measures of image characteristics showed the fingerprints from the contactless scanner make good use of the available grayscale (see 3.2.2.5.1), having entropy values approaching that of inked rolled prints. Signal to noise ratio approximated by edge energy was somewhat lower than that of the contact-based control prints, but not necessarily out of the range observed with inked fingerprints that, typically, have lower edge energy than fingerprints acquired using the Frustrated Total Internal Reflection (FTIR) technology of the Contact Control Scanner used in this study (see 3.2.2.5.2). Spectral analysis indicated ridge spacing characteristics consistent with 500 ppi sampling (see 3.2.2.6). The distributions of all metrics employed showed large dispersions due mainly to the large numbers of fingerprints showing partial or even total loss of ridge detail.

Classification of fingerprints from the contactless scanner using NFIQ shows that fingerprints captured by the contactless scanner are predominantly deemed to be lower in terms of quality classification by NFIQ than those captured by the Contact Control Scanner (see 3.2.3), and these differences are statistically significant at a $\mathrm{p}<0.05$ level.

Matcher testing of fingerprints captured by the contactless scanner showed that while templates generated from the contactless scanner can be compared to templates generated from other images captured by the same scanner, there is a measurable performance reduction when compared to images from the Contact Control Scanner when compared to itself (FNMR of 9.0\% vs. 0.0\%). While an FNMR of 9.0\% may not by itself be telling of significant performance problems, in the case of self-matched images, an FNMR of $9.0 \%$ is indicative of serious problems that cause failure in the trivial case of self-matching. 
This performance degradation was far more pronounced in the mix case of comparing images from the contactless scanner to the Contact Control Scanner where FNMR was measured at $70.0 \%$ indicating that images from the contactless scanner may not be well suited for comparison to legacy images captured by contact-based processes.

\subsubsection{User Satisfaction}

The organization of this section is based on the series of user satisfaction-related questions asked of participants in the post-task questionnaire - specifically, questions 1-5 and 7. It contains both quantitative and qualitative data captured using the questionnaire.

In general, participants found the contact scanner more intuitive, easier to use, faster, and better at providing feedback than the first contactless scanner.

\section{Which of the scanners did you find easier to use? Why?}

Of the 61 participants who interacted with the contactless scanner, 54 said that the contact scanner was easier to use, while only 6 said that the contactless scanner was easier to use; 1 participant said that neither was easier to use.

Table 6: Results for Questionnaire Question 1

\begin{tabular}{c|c|c} 
Contact Scanner & Contactless Scanner & Neither \\
\hline 54 & 6 & 1
\end{tabular}

Participant comments included:

- Hard to align fingers using contactless scanner

- Contact scanner was more "intuitive"

- Contact scanner was faster

\section{Did the scanner lights mean anything to you?}

For the contact scanner, 57 participants in total said that they believed the lights on the device were meant to indicate something to them, while 4 derived no meaning from the lights. For the contactless scanner, only 37 participants said they believed that the lights indicated something: the other 24 said that the lights did not mean anything to them. 
Some participants said that, after completing Task 1 , they did not believe that the lights on the scanner indicated anything, but they changed their minds after seeing the instructional videos. ${ }^{3}$ Six said that the lights had meaning only once they saw the associated video, while only 1 participant said that about the contactless scanner.

A handful of participants who believed that the lights on the scanner were indicative of something provided their interpretations of what those lights were meant to communicate, and failed to interpret them correctly. The number of participants who provided incorrect interpretations of the meaning of the scanner lights for the contact and contactless scanner were 2 and 4, respectively.

Table 7: Results for Questionnaire Question 2

\begin{tabular}{l|c|c|c|c} 
& Yes & No & Yes After Video & Wrong* \\
\hline Contact Scanner & 49 & 4 & 6 & 2 \\
\hline $\begin{array}{l}\text { Contactless } \\
\text { Scanner }\end{array}$ & 32 & 24 & 1 & 4
\end{tabular}

* This means that the participants provided an incorrect interpretation of what the lights on the scanner indicated.

Participant comments included:

- Lights on contact scanner were difficult to notice.

- Thought lights on contactless scanner meant it was warming up

\section{Could you tell what hand the scanner was expecting?}

The majority of the participants in this group - 55 - said that they were able to tell which hand the contact scanner was expecting them to perform a slap with. Only 6 participants said that they were unable to discern which hand the contact scanner was expecting. For the contactless scanner, the numbers were much closer: 34 participants said they could tell which hand the scanner expected them to present, and 23 said they could not tell. Some (4) participants said that the hand expected by the contactless scanner was "not obvious," which is recorded here as being distinct from a "no" response.

\footnotetext{
3 This occurred for other post-task questions to which participants gave yes/no responses: in most cases, at least one participant said that their affirmative response was influenced by the instructional video.
} 
As with responses to the previous question about scanner lights, some participants who answered the question in the affirmative specified they did so because of the instructional video. 6 participants regarding the contact scanner, and 9 regarding the contactless scanner, after seeing the associated instructional videos.

Table 8: Results for Questionnaire Question 3

\begin{tabular}{l|c|c|c|c} 
& Yes & No & Yes After Video & Not Obvious \\
\hline $\begin{array}{l}\text { Contact } \\
\text { Scanner }\end{array}$ & 49 & 6 & 6 & 0 \\
\hline $\begin{array}{l}\text { Contactless } \\
\text { Scanner }\end{array}$ & 25 & 23 & 9 & 4
\end{tabular}

Participant comments included:

- Initially confused until I noticed finger angle in the overlay, even after watching the video (for contactless)

- The left/right templates were ambiguous (for contactless)

\section{Could you tell when the scanner was ready to accept a print?}

Most of the participants (52) said that they could discern when the contact scanner was ready to accept a print -3 said that they were able to do so after watching the instructional video. 9 said that they were unable to do so. For the contactless scanner, almost two-thirds of participants (39) said that they could tell when the scanner was ready to accept a print (5 of them only after seeing the instructional video): 21 said that they could not. One participant said that he/she could "somewhat" tell when the contactless scanner was ready for a print.

Table 9: Results for Questionnaire Question 4

\begin{tabular}{l|c|c|c|c} 
& Yes & No & Yes After Video & Somewhat \\
\hline $\begin{array}{l}\text { Contact } \\
\text { Scanner }\end{array}$ & 49 & 9 & 3 & 0 \\
\hline $\begin{array}{l}\text { Contactless } \\
\text { Scanner }\end{array}$ & 34 & 21 & 5 & 1
\end{tabular}

Participant comments included: 
- Not obvious, ready to scan icon would have helped (for contactless)

- Icon too dim (for contact)

- The hand display prior to the green lights was confusing (for contactless) ${ }^{4}$

\section{Could you tell whether the print was successfully captured?}

58 participants said that they could tell when the contact scanner captured their prints: three said they could not. In contrast, 39 participants said they could tell when the contactless scanner captured their prints ( 3 of them only after seeing the instructional video), while the remaining 22 said they could not.

Table 10: Results for Questionnaire Question 5

\begin{tabular}{l|c|c|c} 
& Yes & No & Yes After Video \\
\hline $\begin{array}{l}\text { Contact } \\
\text { Scanner }\end{array}$ & 58 & 3 & 0 \\
\hline $\begin{array}{l}\text { Contactless } \\
\text { Scanner }\end{array}$ & 36 & 22 & 3
\end{tabular}

Participant comments included:

- Clear sound indication (for contact)

- Couldn't see the boxes were filled (contactless)

- Yes, once it finally was [successfully captured] but not how to get it to accept it What scanner did you prefer? Why?

When asked which scanner they preferred, 54 participants said they preferred the contact scanner: only 6 said that they preferred the contactless scanner. One participant did not favor either one.

\footnotetext{
4 Although few participants commented on this in the questionnaire, many participants voiced this confusion during the test.
} 
Table 11: Results for Questionnaire Question 7

\begin{tabular}{c|c|c} 
Contact & $\begin{array}{c}\text { Contactless } \\
\text { Scanner }\end{array}$ & Both \\
\hline 54 & 6 & 1
\end{tabular}

Participant comments included:

- Placing fingers on a hard surface/glass seems familiar

- Looks a bit "scary" with hardware exposed (for contactless)

- (Contact scanner) was easier to understand and quicker to use.

\subsubsection{Observations}

Participants frequently attempted to touch the glass viewport to leave a fingerprint. In Task 1 - in which participants had no instructions - 38 participants pressed their fingers against the viewport glass and waited for the device to signal a successful capture. Twenty-one of these participants performed their fingerprinting tasks on the contactless scanner first, and so could not have been influenced by using the contact scanner.

Also, the test administrator observed that many participants had difficulty with the positioning cues (see Figure 4) of the contactless scanner, especially before they viewed the instructional video. During Task 1, only 11 out of 61 participants had their prints successfully captured by the contactless scanner. In Task 2, after the participants had viewed the instructional video for the contactless scanner, 34 participants were successful and 27 were not.

Shorter participants had difficulty looking into the viewport and seeing the virtual overlay on the contactless scanner. The virtual overlay was created by a LED screen being reflected from a beam splitter (see Fig. 12). 


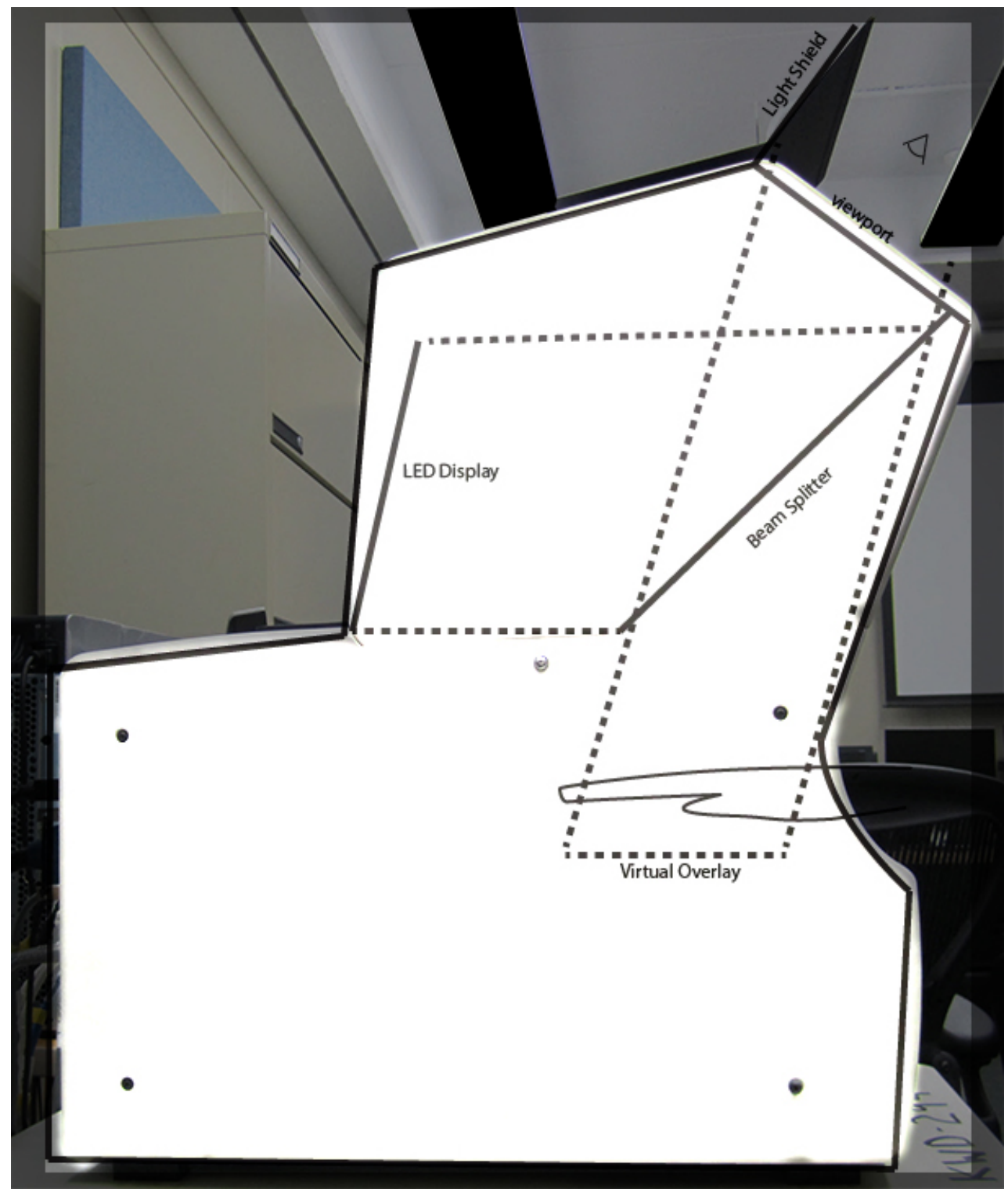

Figure 24: Virtual Overlay Mechanics

When viewed through the viewport at the appropriate angle, the beam splitter enabled participants to see both the reflection of the LED screen and their hand, so that the outline of the hand shown on the LED screen appeared to be superimposed on the participant's hand. The superimposition of the hand overlay could only be seen if the participant could look down into the viewport. Participants whose eyes were closer to the level of the viewport had difficulty achieving the appropriate viewing angle. 


\section{DISCUSSION}

The results of this study indicate that while contactless fingerprinting technology is viable the prototype consistently functioned as intended when used correctly - it presents a number of usability challenges.

\subsection{MENTAL MODELS OF FINGERPRINTING - TOUCHING THE GLASS}

During Task 1, when they were working without instructions, many participants placed their hands on the viewport glass of the contactless scanner . As noted in Sec. 3.2.7, participants very frequently did this regardless of whether or not they used the contact scanner before using the contactless scanner. This indicates that people's mental model (or at least the participants' mental model) of fingerprint collection involves pressing their fingers against a surface. Touching a glass surface seemed to be deeply ingrained into the study participants' idea of what fingerprint collection entails - a perception that may be influenced by depictions of fingerprint scanning in popular media and past experiences with electronic fingerprinting.

Some adjustments could be made to the contactless scanner to help counteract this erroneous mental model, specifically by increasing the affordance of its viewport. As one participant suggested, an effective approach might be "chang[ing] the glass so that it looks like goggles so people know they are supposed to look through the glass and don't put their hands on it."

\subsection{FINGERPRINTING SEQUENCE CUES}

A usability challenge common to fingerprint scanners of any type - including the contactless scanner used in this study - is that of informing the user where they are in the fingerprinting process (see Figure 8 for the fingerprinting task timeline). A fingerprint collection device needs to clearly indicate:

1. readiness to accept prints;

2. which hand is expected (right or left);

3. appropriate hand/finger placement;

4. successful print acquisition; and

5. how to recover from a failed attempt.

The contactless scanner indicated readiness to accept prints by illuminating green LEDs inside the case where the participants were to place their hands, as seen in Figure 25. A number of participants did not realize that these LEDs indicated anything (see Table 4). 
Even those who recognized the lights as an indicator may have misinterpreted them: for example, one participant said that the "lights come on and I think it is just warming up."

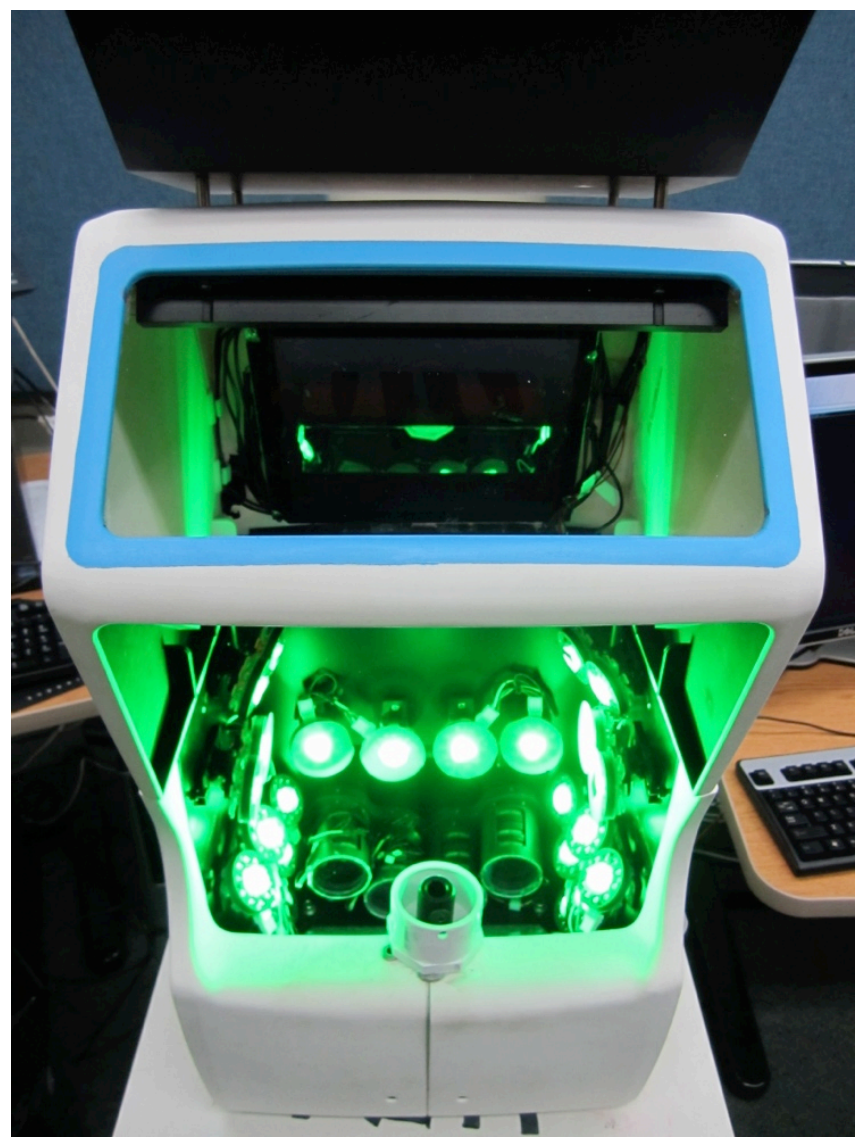

Figure 25: Ready State LEDs

\subsection{HAND INDICATION (RIGHT OR LEFT)}

The contactless scanner indicated which hand it expected using a virtual overlay (seen in Figure 26 below). This indicator proved sufficient for most participants, once they knew to look into the scanner viewport. However, the fact that the contactless scanner fingerprinting process started with the left hand made it counterintuitive to some participants. 


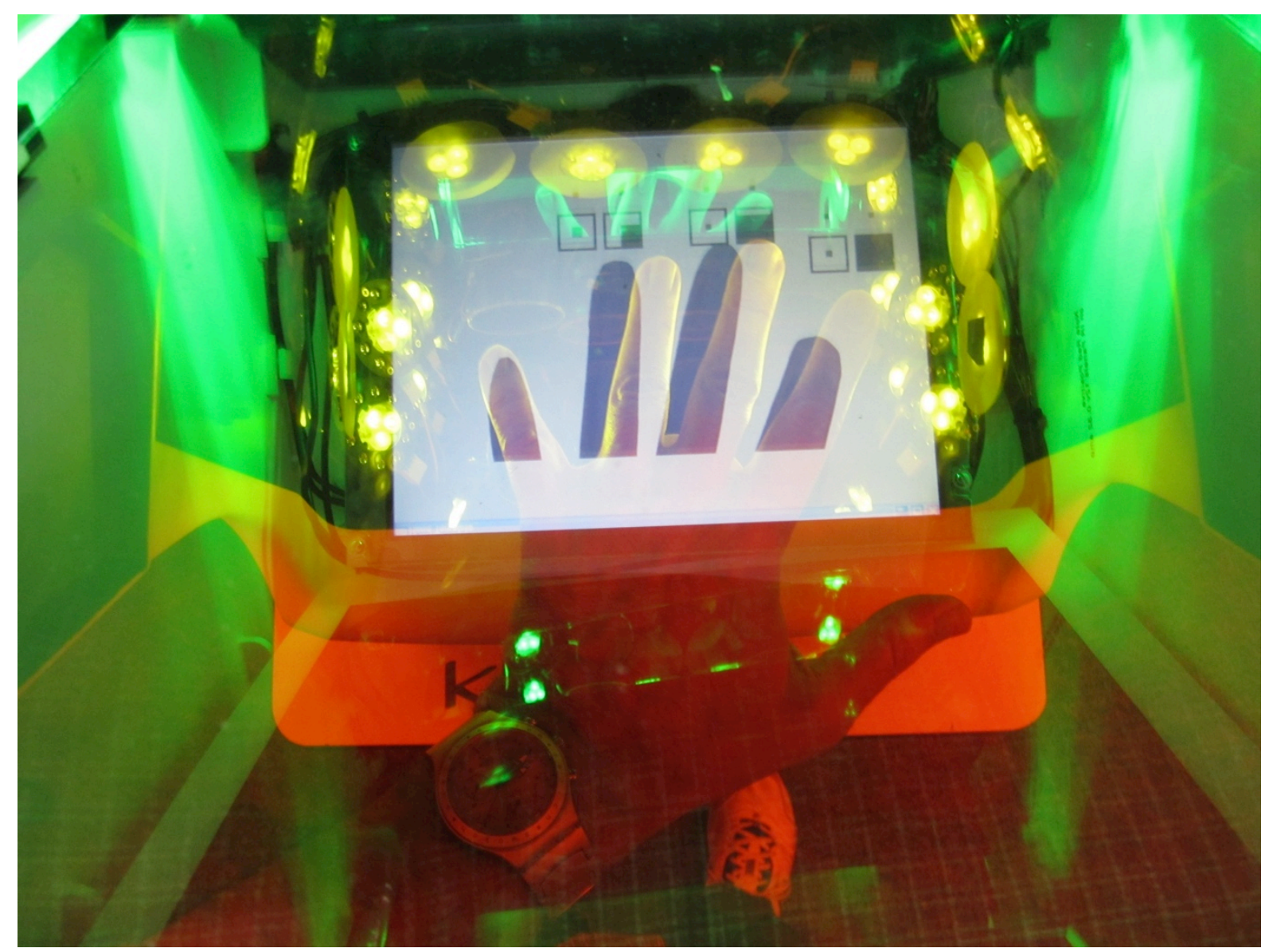

Figure 26: Virtual Overlay as Seen Through Viewport

\subsection{FINGER PLACEMENT INDICATORS}

During the fingerprint collection process with the contactless scanner, some participants remarked on how difficult it was to coordinate the filling of the squares based on good hand placement (see Figure 4). As one square filled with an up/down movement and the other square filled with left/right, forward/back movements, participants had difficulty moving a finger to fill one box without affecting the other box (or boxes associated with the other fingers).

An additional aspect of the positioning cues that proved to be challenging was the signaling of a captured print. When a print was successfully captured, the positioning cues associated with the print would be replaced with an oval in a box for an instant before all cues for that finger were removed from display. Unfortunately, the positioning cues would also disappear when the associated finger went outside the tracking area. This made it easy for participants to mistake the scanner’s “missing” a finger for a successful print capture. 


\section{CONCLUSIONS}

The persistent mental model of an electronic fingerprinting process requiring the user to touch a glass surface has two major implications for DHS if the organization plans to continue developing contactless fingerprint technology. First, a concerted effort needs to be made to educate the public in the use of contactless scanners. This means using a number of different media - such as video, posters, and Web information - to communicate the same message about how contactless scanners work and how to operate them.

Second, it should be accepted, and planned for, that people will touch any clear, flat surface on a contactless fingerprint scanner and that surface will need to be cleaned. During pilot testing of the scanners prior to the usability study, the research team discovered that it did not take long for the latent prints left on the contactless scanner glass surface to interfere with the collection of live prints. ${ }^{5}$ This means that in any operational concept of the scanner tested during this study, there will have to be some procedures to clean them.

Educating the public on the proper way to use contactless scanners and keeping the glass surfaces clean enough to capture prints will take time. This time will have to be accounted for when planning throughput numbers for any fingerprinting process involving contactless scanners. It is likely that while people are still growing accustomed to contactless scanners, it will take longer to successfully collect fingerprints, leading to longer lines at entry points.

\footnotetext{
5 This did not present as much of a problem during actual testing, as all fingerprinting devices were cleaned so as not to leave any prints that might serve as cues of what to do with the scanner.
} 


\section{REFERENCES}

[1] “Advanced Data From Vital and Health Statistics”, US Department of Health and Human Services Centers for Disease Control and Prevention, October 27, 2004, http://www.cdc.gov/nchs/data/ad/ad347.pdf

[2] Tabassi, E., Wilson, C., \& Watson, C. U.S. Department of Commerce, National Institute of Standards and Technology (NIST). (2004). Fingerprint image quality (NIST IR 7151). Retrieved from http://www.nist.gov/manuscript-publicationsearch.cfm?pub_id=905710

[3] Theofanos, M., Orandi, S., Micheals, R., Stanton, B., \& Zhang, N. F. Department of Commerce, National Institute of Standards and Technology (NIST). (2007). Effects of scanner height on fingerprint capture (NIST IR 7382). Retrieved from http://www.nist.gov/manuscript-publication-search.cfm?pub_id=50903

[4] Theofanos, M., Stanton, B., Orandi, S., Micheals, R., \& Zhang, N. F. Department of Commerce, National Institute of Standards and Technology (NIST). (2006). Usability testing of ten-print fingerprint capture (NIST IR 7403). Retrieved from http://www.nist.gov/manuscript-publication-search.cfm?pub_id=51123

[5] ISO 9241-210:2010 Ergonomics of human-system interaction - Part 210: Humancentred design for interactive systems

[6] Tukey, J. W. Exploratory Data Analysis. Addison-Wesley, Reading. 1977.

[7] Zhang, Z. and Blum, R. S. On estimating the quality of noisy images, Proceedings of the 1998 IEEE International Conference on Acoustics, Speech and Signal Processing, Vol. 5, 1998, Page(s): 2897 - 2900.

[8] Libert, J. M., Orandi, S., Grantham, J. A 1D Spectral Image Validation/Verification Metric for Fingerprints (NIST IR 7599), 2009, http://nvlpubs.nist.gov/nistpubs/ir/2009/ir7599.pdf .

[9] Wilcoxon, Frank . Individual comparisons by ranking methods. Biometrics Bulletin 1 (6), (1945), 80-83.

[10] Wright, P, S. P-values for simultaneous inference. Biometrics, Vol. 48, No. 4 ( 1992), pp. 1005-1013. 


\section{APPENDIX A: DEMOGRAPHICS QUESTIONNAIRE \\ National Institute of Standards and Technology (NIST) \\ Information Technology Laboratory (ITL), Information Access Division (IAD) \\ Contactless Fingerprint Capture Study Task Evaluation}

\section{Demographic Questionnaire}

1. Age:

2. Gender:

Female __ Male

3. Handedness: __ Right handed

4. Height: 
APPENDIX B: POST-TASK QUESTIONNAIRE

\author{
National Institute of Standards and Technology (NIST) \\ Information Technology Laboratory (ITL), Information Access Division (IAD) \\ Contactless Fingerprint Capture Study Task Evaluation Post-Task Questionnaire
}

Based on your experience from the tasks with the fingerprint scanners, please answer the following questions.

1. Which of the scanners did you find easier to use? Why?

2. Did the lights on the scanners mean anything to you?

a. Scanner A: 
b. Scanner B:

3. Could you tell what hand the scanner was expecting?

a. Scanner A:

b. Scanner B:

4. Could you tell when the scanner was ready to accept a print?

a. Scanner A: 
b. Scanner B:

5. Could you tell whether the print was successfully captured?

a. Scanner A:

b. Scanner B: 
6. What improvements would you make to the scanners? Why?

a. Scanner A:

b. Scanner B:

7. What scanner did you prefer? Why? 
Do you have any additional comments? (Do not include any personally identifiable information in your comments) 